INSTITUT NATIONAL DE RECHERCHE EN INFORMATIQUE ET EN AUTOMATIQUE

\title{
Mathematical Modeling of Electrocardiograms: A Numerical Study
}

\author{
Muriel Boulakia — Serge Cazeau — Miguel A. Fernández \\ Jean-Frédéric Gerbeau — Nejib Zemzemi
}

\section{$\mathbf{N}^{\circ} 6977$}

June 2009

Thème BIO

apport

derecherche 



\title{
RINRIA
}

\section{Mathematical Modeling of Electrocardiograms: A Numerical Study}

\author{
Muriel Boulakia刚, Serge Cazeau用, Miguel A. Fernández ${ }^{\dagger}$

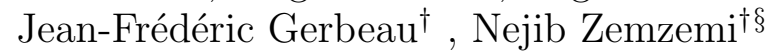 \\ Thème BIO - Systèmes biologiques \\ Projet REO
}

Rapport de recherche $n^{\circ} 6977$ - June 2009 - 42 pages

\begin{abstract}
This report deals with the numerical simulation of electrocardiograms (ECG). Our aim is to devise a mathematical model, based on partial differential equations, which is able to provide realistic 12-lead ECGs. The main ingredients of this model are classical: the bidomain equations coupled to a phenomenological ionic model in the heart, and a generalized Laplace equation in the torso. The obtention of realistic ECGs relies on other important features - including heart-torso transmission conditions, anisotropy, cell heterogeneity and His bundle modeling - that are discussed in detail. The numerical implementation is based on state-of-the-art numerical methods: domain decomposition techniques and second order semi-implicit time marching schemes, offering a good compromise between accuracy, stability and efficiency. The numerical ECGs obtained with this approach show correct amplitudes, shapes and polarities, in all the 12 standard leads. The relevance of every modeling choice is carefully discussed and the numerical ECG sensitivity to the model parameters investigated.
\end{abstract}

Key-words: 12-lead electrocardiogram, cardiac electrophysiology, mathematical modeling, numerical simulation, bidomain equation, ionic model, heart-torso coupling, monodomain equation, sensitivity analysis.

\footnotetext{
* Université Paris 6, Laboratoire Jacques-Louis Lions, F-75005 Paris, France

$\dagger$ INRIA, REO project-team

¥ Département de Rythmologie-Stimulation, Hôpital Saint-Joseph, 185, rue Raymond Losserand, F-75014 Paris, France

$\S$ Université Paris 11, Laboratoire de mathématiques d'Orsay, F-91405 Orsay Cedex,
} France

Unité de recherche INRIA Rocquencourt

Domaine de Voluceau, Rocquencourt, BP 105, 78153 Le Chesnay Cedex (France)

Téléphone : +33139635511-Télécopie : +33139635330 


\section{Modélisation mathématique de l'électrocardiogramme : une étude numérique}

Résumé : Dans ce rapport nous abordons la simulation numérique de l'électrocardiogramme (ECG). Let but est de proposer un modèle mathématique, basé sur des équations aux dérivées partielles, capable de simuler des ECGs (à 12 dérivations) réalistes. Les ingrédients de ce modèlse sont classiques: équation bidomaine couplée avec un modèle ionique phénoménologique dans le cœur, et équation de Laplace généralisée dans le torse. L'obtention d'ECGs rélalistés repose sur d'autres caractéristiques importantes (telles que les conditions de couplage cœur-torse, l'anisotropie, l'hétérogénité cellulaire et le faisceau de His) qui sont discutées en détail. La mise en œuvre numérique est basée sur des algorithmes dans l'état de l'art du calcul scientifique: méthodes de décomposition de domaine et schémas de marche en temps semi-implicites d'ordre deux, offrant un bon compromis entre précision, stabilité et éfficacité. Les ECGs numériques obtenus avec cette approche ont des amplitudes, formes et polarités physiologiques dans les 12 dérivations. La pertinence de chaque choix de modélisation est soigneusement discuté. Cette étude comporte aussi une analyse de sensibilité de l'ECG numérique par rapport aux paramètres du modèle.

Mots-clés : Électrocardiogramme à 12 dérivations, électrophysiologie cardiaque, modélisation mathématique, simulation numérique, équation bidomaine, modèle ionique, couplage cœur-torse, équation monodomaine, analyse de sensibilité. 


\section{Introduction}

The electrocardiogram (ECG) is a noninvasive recording of the electrical activity of the heart, obtained from a standard set of skin electrodes and presented to the physician as the "12-lead ECG": that is, 12 graphs of the recorded voltage vs. time. The ECG can be considered as the most widely used clinical tool for the detection and diagnosis of a broad range of cardiac conditions (see e.g. [1, 24]). Despite that, the clinical significance of some ECG findings is still not fully understood. Computer based simulations of the ECG, linking models of the electrical activity of the heart (in normal or pathological condition) to the ECG signal, can therefore be a valuable tool for improving this knowledge. Such an ECG simulator can also be useful in building a virtual data base of pathological conditions, in order to test and train medical devices [16]. Moreover, being able to simulate realistic ECGs is a necessary step toward the development of patient-specific models from clinical ECG data.

The mathematical modeling of the ECG is known as the forward problem of electrocardiography [32]. It relies on three main ingredients: a model for the electrical activity of the heart, a model for the torso (extracardiac regions) and some specific heart-torso coupling conditions. Within each of these components, several options are possible, with different levels of complexity and realism (see 32 for a recent comprehensive review).

Although many works have been devoted to the numerical simulation of cardiac electrophysiology (see e.g. the monographs [47, 44, 51 and the references therein), only a small number [28, 41, 32, 30, 54, 43. addresses the numerical simulation of ECGs using a whole-heart reaction-diffusion (i.e. bidomain or monodomain) model. Among them, only a very few 41, 43. provide meaningful simulations of the complete 12-lead ECG. These simulations rely on a monodomain description of the electrical activity the heart, a decoupling of the heart and the torso (isolated heart assumption) and a multi-dipole approximation of the cardiac source within the torso (see [32, Section 4.2.4] and [26]). To the best of our knowledge, none of the existing approaches based on partial differential equations (PDE) and a fully coupled heart-torso formulation (see e.g. [32, Section 4.6] and [51) have shown realistic 12-lead ECG simulations.

The main ingredients of our mathematical ECG model are standard (see e.g. [44, 51, 32]): bidomain equations and phenomenological cell model for the heart, and a generalized Laplace equation for the torso. Nevertheless, once these ingredients have been chosen, several other critical aspects have to be elucidated: heart-torso transmission conditions, cell heterogeneity, His bundle modeling, anisotropy, etc.

The purpose of the present work is therefore twofold: first, provide realistic simulations of the 12-lead ECG based on a complete PDE model with a fully coupled heart-torso formulation; second, discuss through numerical simulations the impact of various modeling options and the sensitivity to the model parameters. Note that the achievement of these two goals is a fundamental step prior to addressing the inverse problem of electrocardiography, which consists in identifying the ECG model parameters from clinical ECG data.

The numerical methods proposed to solve the problem offer a good balance between efficiency, stability and accuracy. The PDE system made of the heart and torso models is solved using a finite element method and a second order semi-implicit time marching scheme (see e.g. [45]). The coupling conditions at 
the heart-torso interface are enforced by a Dirichlet-Neumann domain decomposition algorithm (see e.g. [46, 53]).

The remainder of the this paper is organized as follows. The ECG model equations are presented in section 2 Section 3 is devoted to the description of the numerical algorithm. The numerical ECGs obtained with the resulting computational model, under a healthy and a pathological (bundle branch block) condition, are presented and discussed in section 4. Section 5 investigates the impact, on the ECG, of various modeling assumptions: heart-torso uncoupling, monodomain approximation, isotropy, cell homogeneity, resistance-capacitance behavior of the pericardium. In section 6, we present a time and space convergence study in terms of the ECG. The sensitivity of the ECG to the main model parameters is also investigated. At last, conclusions and some lines of forthcoming research are drawn in section 7 .

\section{Modeling}

This section contains standard material (see e.g. [51, Chapter 2]). It introduces notation and the coupled system of partial and ordinary differential equations (PDE/ODE) involved in the reference mathematical model considered in this paper.

\subsection{Heart tissue}

Our reference model for the electrical activity of the heart is the so-called bidomain model [55, 51, 44]. This macroscopic model is based on the assumption that, at the cell scale, the cardiac tissue can be viewed as partitioned into two ohmic conducting media, separated by the cell membrane: intracellular, made of the cardiac cells, and extracellular which represents the space between them. After an homogenization process (see [37, 39]), the intra- and extracellular domains can be supposed to occupy the whole heart volume $\Omega_{\mathrm{H}}$ (this also applies to the cell membrane). Hence, the averaged intra- and extracellular densities of current, $\boldsymbol{j}_{\mathrm{i}}$ and $\boldsymbol{j}_{\mathrm{e}}$, conductivity tensors, $\boldsymbol{\sigma}_{\mathrm{i}}$ and $\boldsymbol{\sigma}_{\mathrm{e}}$, and electric potentials, $u_{\mathrm{i}}$ and $u_{\mathrm{e}}$, are defined in $\Omega_{\mathrm{H}}$. The electrical charge conservation becomes

$$
\operatorname{div}\left(\boldsymbol{j}_{\mathrm{i}}+\boldsymbol{j}_{\mathrm{e}}\right)=0, \quad \text { in } \Omega_{\mathrm{H}},
$$

and the homogenized equation of the electrical activity of the cell membrane is given by

$$
A_{\mathrm{m}}\left(C_{\mathrm{m}} \frac{\partial V_{\mathrm{m}}}{\partial t}+I_{\mathrm{ion}}\left(V_{\mathrm{m}}, w\right)\right)+\operatorname{div}\left(j_{\mathrm{i}}\right)=A_{\mathrm{m}} I_{\mathrm{app}}, \quad \text { in } \quad \Omega_{\mathrm{H}},
$$

complemented with the Ohm's laws

$$
j_{\mathrm{i}}=-\sigma_{\mathrm{i}} \boldsymbol{\nabla} u_{\mathrm{i}}, \quad j_{\mathrm{e}}=-\sigma_{\mathrm{e}} \boldsymbol{\nabla} u_{\mathrm{e}} .
$$

Here, $V_{\mathrm{m}}$ stands for the transmembrane potential, defined as

$$
V_{\mathrm{m}} \stackrel{\text { def }}{=} u_{\mathrm{i}}-u_{\mathrm{e}},
$$

$A_{\mathrm{m}}$ is a constant representing the rate of membrane area per volume unit and $C_{\mathrm{m}}$ the membrane capacitance per area unit. The term $I_{\mathrm{ion}}\left(V_{\mathrm{m}}, w\right)$ represents 
the ionic current across the membrane and $I_{\text {app }}$ a given applied current stimulus. Both currents are measured per membrane area unit.

In general, the ionic variable $w$ (possibly vector valued) satisfies a system of ODE of the type:

$$
\frac{\partial w}{\partial t}+g\left(V_{\mathrm{m}}, w\right)=0, \quad \text { in } \quad \Omega_{\mathrm{H}} .
$$

The definition of the functions $g$ and $I_{\text {ion }}$ depends on the considered cell ionic model (see [55, 51, 44] and the references therein). According to their degree of complexity and realism, the ionic models typically fall into one of the following categories (see [44, Chapter 3]): phenomenological (e.g. [19, [56, 18, 36]) or physiological (e.g. [4, 34, 33, 38, 15]).

In this study, the phenomenological two-variable model proposed by Mitchell and Schaeffer in [36] is considered (rescaled version). The functions $g$ and $I_{\text {ion }}$ are then given by

$$
\begin{gathered}
I_{\text {ion }}\left(V_{\mathrm{m}}, w\right)=-\frac{w}{\tau_{\mathrm{in}}} \frac{\left(V_{\mathrm{m}}-V_{\min }\right)^{2}\left(V_{\mathrm{max}}-V_{\mathrm{m}}\right)}{V_{\max }-V_{\min }}+\frac{1}{\tau_{\text {out }}} \frac{V_{\mathrm{m}}-V_{\min }}{V_{\max }-V_{\min }}, \\
g\left(V_{\mathrm{m}}, w\right)=\left\{\begin{array}{rr}
\frac{w}{\tau_{\text {open }}}-\frac{1}{\tau_{\text {open }}\left(V_{\max }-V_{\min }\right)^{2}} & \text { if } V_{\mathrm{m}}<V_{\text {gate }}, \\
\frac{w}{\tau_{\text {close }}} & \text { if } \quad V_{\mathrm{m}}>V_{\text {gate }},
\end{array}\right.
\end{gathered}
$$

where $\tau_{\text {in }}, \tau_{\text {out }}, \tau_{\text {open }}, \tau_{\text {close }}, V_{\text {gate }}$ are given parameters and $V_{\text {min }}, V_{\text {max }}$ scaling constants (typically -80 and $20 \mathrm{mV}$, respectively).

Despite its reduced complexity (2 state variables, 5 free parameters), the Mitchell-Schaeffer model integrates relevant physiological properties of the cell membrane: transmembrane potential, activation dynamics and two currents (inward and outward) leading to depolarization and repolarization. Moreover, owing to its planar character, the model can be understood analytically (see e.g. 36]), which allows to identify how the free parameters affect its behavior (see subsection 4.1.5).

The gate variable $w$ depends on the change-over voltage $V_{\text {gate }}$ and on the time constants for opening, $\tau_{\text {open }}$, and closing, $\tau_{\text {close }}$. The time constants $\tau_{\text {in }}$ and $\tau_{\text {close }}$ are respectively related to the length of the depolarization and repolarization (final stage) phases. Typically, these constants are such that $\tau_{\text {in }} \ll \tau_{\text {out }} \ll$ $\tau_{\text {open }}, \tau_{\text {close }}$.

To sum up, the system of equations modeling the electrical activity within the heart is

$$
\left\{\begin{array}{r}
A_{\mathrm{m}}\left(C_{\mathrm{m}} \frac{\partial V_{\mathrm{m}}}{\partial t}+I_{\mathrm{ion}}\left(V_{\mathrm{m}}, w\right)\right)-\operatorname{div}\left(\boldsymbol{\sigma}_{\mathrm{i}} \boldsymbol{\nabla} V_{\mathrm{m}}\right)-\operatorname{div}\left(\boldsymbol{\sigma}_{\mathrm{i}} \boldsymbol{\nabla} u_{\mathrm{e}}\right)=A_{\mathrm{m}} I_{\mathrm{app}}, \quad \text { in } \Omega_{\mathrm{H}}, \\
-\operatorname{div}\left(\left(\boldsymbol{\sigma}_{\mathrm{i}}+\boldsymbol{\sigma}_{\mathrm{e}}\right) \boldsymbol{\nabla} u_{\mathrm{e}}\right)-\operatorname{div}\left(\boldsymbol{\sigma}_{\mathrm{i}} \boldsymbol{\nabla} V_{\mathrm{m}}\right)=0, \\
\frac{\partial w}{\partial t}+g\left(V_{\mathrm{m}}, w\right)=0, \quad \text { in } \Omega_{\mathrm{H}},
\end{array}\right.
$$

with $g$ and $I_{\text {ion }}$ given by (2.6). This system has to be complemented with appropriate initial and boundary conditions. Denoting by $V_{\mathrm{m}}^{0}$ and $w^{0}$ given initial data for the transmembrane potential and the gate variable, the following initial condition must be enforced

$$
V_{\mathrm{m}}(\boldsymbol{x}, 0)=V_{\mathrm{m}}^{0}(\boldsymbol{x}), \quad w(\boldsymbol{x}, 0)=w^{0}(\boldsymbol{x}) \quad \forall \boldsymbol{x} \in \Omega_{\mathrm{H}} .
$$


As regards the boundary conditions on $\Sigma \stackrel{\text { def }}{=} \partial \Omega_{\mathrm{H}}$ (see Figure 11 , it is widely assumed (see e.g. [55, 31, 44, 51]) that the intracellular current does not propagate outside the heart. Consequently,

$$
\boldsymbol{j}_{\mathrm{i}} \cdot \boldsymbol{n}=\boldsymbol{\sigma}_{\mathrm{i}} \boldsymbol{\nabla} u_{\mathrm{i}} \cdot \boldsymbol{n}=0, \quad \text { on } \quad \Sigma,
$$

where $\boldsymbol{n}$ stands for the outward unit normal to $\Omega_{\mathrm{H}}$. Equivalently, and owing to the divergence structure of 2.7$]_{1}$, this condition can be enforced as

$$
\boldsymbol{\sigma}_{\mathrm{i}} \boldsymbol{\nabla} V_{\mathrm{m}} \cdot \boldsymbol{n}+\boldsymbol{\sigma}_{\mathrm{i}} \boldsymbol{\nabla} u_{\mathrm{e}} \cdot \boldsymbol{n}=0, \quad \text { on } \quad \Sigma .
$$

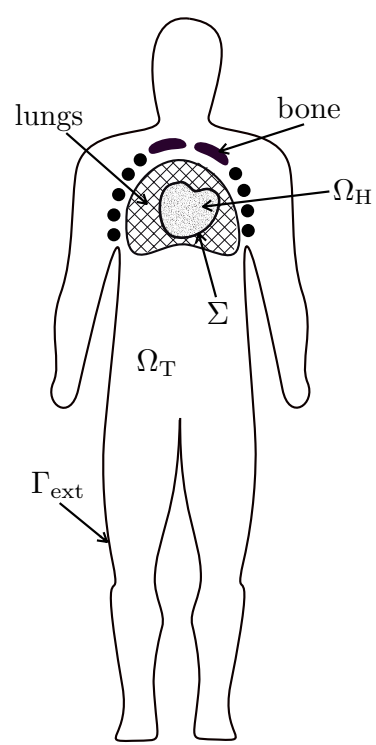

Figure 1: Geometry description: the heart domain $\Omega_{\mathrm{H}}$ and the torso domain $\Omega_{\mathrm{T}}$ (extramyocardial regions)

\subsection{Coupling with torso}

To set up boundary conditions on the extracellular potential $u_{\mathrm{e}}$, a perfect electric transmission between the heart and the torso domains is generally assumed (see e.g. [55, 31, 44, 51]):

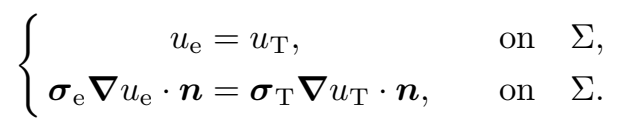

Here, $u_{\mathrm{T}}$ and $\boldsymbol{\sigma}_{\mathrm{T}}$ stand respectively for the potential and conductivity tensor of the torso tissue, denoted by $\Omega_{\mathrm{T}}$ (see Figure 1). Note that, with (2.9), the current continuity condition $(2.10)_{2}$ is consistent with the divergence structure of 2.7$)_{2}$. Other possible heart-torso transmission conditions will be discussed in sections 5.1 and 5.5 
Under the quasi-static assumption [35, the torso can be viewed as a passive conductor. Therefore, the potential $u_{\mathrm{T}}$ satisfies the generalized Laplace equation:

$$
\operatorname{div}\left(\boldsymbol{\sigma}_{\mathrm{T}} \boldsymbol{\nabla} u_{\mathrm{T}}\right)=0, \quad \text { in } \quad \Omega_{\mathrm{T}} .
$$

This equation is complemented with a boundary condition on the external boundary $\Gamma_{\text {ext }} \stackrel{\text { def }}{=} \partial \Omega_{\mathrm{T}} \backslash \Sigma$ (see Figure 1). Moreover, assuming that no current can flow from the torso across $\Gamma_{\text {ext }}$, we enforce

$$
\boldsymbol{\sigma}_{\mathrm{T}} \boldsymbol{\nabla} u_{\mathrm{T}} \cdot \boldsymbol{n}_{\mathrm{T}}=0, \quad \text { on } \quad \Gamma_{\text {ext }},
$$

where $\boldsymbol{n}_{\mathrm{T}}$ stands for the outward unit normal to $\Omega_{\mathrm{T}}$.

In summary, our reference model for the ECG is based on the coupled solution of systems (2.7), (2.6) and (2.11), completed with the boundary conditions (2.9) and 2.12), the interface conditions (2.10) and the initial condition 2.8). Throughout this study, this system of equations will be termed RM (reference model), which is also known in the literature as full bidomain model (see e.g. 9]). The interested reader is referred to [7 for a recent study on the mathematical well-posedness of this system, under appropriate assumptions on the structure of $I_{\text {ion }}$ and $g$.

Although additional complexity and realism can still be introduced through the ionic model (see e.g. 44, 34, 33, 38, 15]), this coupled system can be considered as the state-of-the-art in the PDE/ODE modeling of the ECG (see e.g. [32]).

\section{$3 \quad$ Numerical methods}

This section is devoted to a brief presentation of he numerical method used to solve the coupled problem RM.

\subsection{Space and time discretization}

The discretization in space is performed by applying the finite element method to an appropriate weak formulation of this coupled problem. Let $\Omega$ be the interior of $\bar{\Omega}_{\mathrm{H}} \cup \bar{\Omega}_{\mathrm{T}}$. Problem $\mathbf{R M}$ can be rewritten in weak form as follows (see e.g. [7): for $t>0, V_{\mathrm{m}}(\cdot, t) \in H^{1}\left(\Omega_{\mathrm{H}}\right), w(\cdot, t) \in L^{\infty}\left(\Omega_{\mathrm{H}}\right)$ and $u(\cdot, t) \in H^{1}(\Omega)$, with $\int_{\Omega_{\mathrm{H}}} u=0$, such that

$$
\left\{\begin{array}{l}
A_{\mathrm{m}} \int_{\Omega_{\mathrm{H}}}\left(C_{\mathrm{m}} \frac{\partial V_{\mathrm{m}}}{\partial t}+I_{\mathrm{ion}}\left(V_{\mathrm{m}}, w\right)\right) \phi+\int_{\Omega_{\mathrm{H}}} \boldsymbol{\sigma}_{\mathrm{i}} \boldsymbol{\nabla}\left(V_{\mathrm{m}}+u\right) \cdot \boldsymbol{\nabla} \phi=A_{\mathrm{m}} \int_{\Omega_{\mathrm{H}}} I_{\mathrm{app}} \phi, \\
\int_{\Omega_{\mathrm{H}}}\left(\boldsymbol{\sigma}_{\mathrm{i}}+\boldsymbol{\sigma}_{\mathrm{e}}\right) \boldsymbol{\nabla} u \cdot \boldsymbol{\nabla} \psi+\int_{\Omega_{\mathrm{H}}} \boldsymbol{\sigma}_{\mathrm{i}} \boldsymbol{\nabla} V_{\mathrm{m}} \cdot \boldsymbol{\nabla} \psi+\int_{\Omega_{\mathrm{T}}} \boldsymbol{\sigma}_{\mathrm{T}} \boldsymbol{\nabla} u \cdot \nabla \psi=0, \\
\frac{\partial w}{\partial t}+g\left(V_{\mathrm{m}}, w\right)=0, \quad \text { in } \Omega_{\mathrm{H}},
\end{array}\right.
$$

for all $(\phi, \psi) \in H^{1}\left(\Omega_{\mathrm{H}}\right) \times H^{1}(\Omega)$, with $\int_{\Omega_{\mathrm{H}}} \psi=0$. The potentials in the heart and the torso are recovered by setting $u_{\mathrm{e}}=u_{\mid \Omega_{\mathrm{H}}}$ and $u_{\mathrm{T}}=u_{\mid \Omega_{\mathrm{T}}}$. Note that this weak formulation (3.13) integrates, in a natural way, the coupling conditions 2.10 . 
The space semi-discretized formulation is based on $\sqrt{3.13}$ and obtained by replacing the functional spaces by finite dimensional spaces of continuous piecewise affine functions, $V_{h} \subset H^{1}\left(\Omega_{\mathrm{H}}\right)$ and $W_{h} \subset H^{1}(\Omega)$.

The resulting system is discretized in time by combining a second order implicit scheme (backward differentiation formulae, see e.g. 45]) with an explicit treatment of the ionic current. We refer to [17] for a recent review which suggests the use of second order schemes. Let $N \in \mathbb{N}^{*}$ be a given integer and consider a uniform partition $\left\{\left[t_{n}, t_{n+1}\right]\right\}_{0 \leq n \leq N-1}$, with $t_{n} \stackrel{\text { def }}{=} n \delta t$, of the time interval of interest $[0, T]$, with a time-step $\delta t \stackrel{\text { def }}{=} T / N$. Denote by $\left(V_{\mathrm{m}}^{n}, u^{n}, w^{n}\right)$ the approximated solution obtained at time $t_{n}$. Then, $\left(V_{\mathrm{m}}^{n+1}, u^{n+1}, w^{n+1}\right)$ is computed as follows: For $0 \leq n \leq N-1$

1. Second order extrapolation: $\widetilde{V}_{\mathrm{m}}^{n+1} \stackrel{\text { def }}{=} 2 V_{\mathrm{m}}^{n}-V_{\mathrm{m}}^{n-1}$;

2. Solve for $w^{n+1} \in V_{h}$ :

$$
\frac{1}{\delta t}\left(\frac{3}{2} w^{n+1}-2 w^{n}+\frac{1}{2} w^{n-1}\right)+g\left(\widetilde{V}_{\mathrm{m}}^{n+1}, w^{n+1}\right)=0, \quad \text { (nodal-wise) }
$$

3. Ionic current evaluation: $I_{\mathrm{ion}}\left(\widetilde{V}_{\mathrm{m}}^{n+1}, w^{n+1}\right)$;

4. Solve for $\left(V_{\mathrm{m}}^{n+1}, u^{n+1}\right) \in V_{h} \times W_{h}$, with $\int_{\Omega_{\mathrm{H}}} u^{n+1}=0$ :

$$
\left\{\begin{array}{c}
A_{\mathrm{m}} \int_{\Omega_{\mathrm{H}}} \frac{C_{\mathrm{m}}}{\delta t}\left(\frac{3}{2} V_{\mathrm{m}}^{n+1}-2 V_{\mathrm{m}}^{n}+\frac{1}{2} V_{\mathrm{m}}^{n-1}\right) \phi+\int_{\Omega_{\mathrm{H}}} \boldsymbol{\sigma}_{\mathrm{i}} \nabla\left(V_{\mathrm{m}}^{n+1}+u^{n+1}\right) \cdot \nabla \phi \\
=A_{\mathrm{m}} \int_{\Omega_{\mathrm{H}}}\left(I_{\mathrm{app}}\left(t_{n+1}\right)-I_{\mathrm{ion}}\left(\widetilde{V}_{\mathrm{m}}^{n+1}, w^{n+1}\right)\right) \phi \\
\int_{\Omega_{\mathrm{H}}}\left(\boldsymbol{\sigma}_{\mathrm{i}}+\boldsymbol{\sigma}_{\mathrm{e}}\right) \nabla u^{n+1} \cdot \nabla \psi+\int_{\Omega_{\mathrm{H}}} \boldsymbol{\sigma}_{\mathrm{i}} \nabla V_{\mathrm{m}}^{n+1} \cdot \nabla \psi+\int_{\Omega_{\mathrm{T}}} \boldsymbol{\sigma}_{\mathrm{T}} \boldsymbol{\nabla} u^{n+1} \cdot \nabla \psi=0
\end{array}\right.
$$

for all $(\phi, \psi) \in V_{h} \times W_{h}$, with $\int_{\Omega_{\mathrm{H}}} \psi=0$. Finally, set $u_{\mathrm{e}}^{n+1}=u_{\mid \Omega_{\mathrm{H}}}^{n+1}$ and $u_{\mathrm{T}}^{n+1}=u_{\mid \Omega_{\mathrm{T}}}^{n+1}$.

The above algorithm is semi-implicit (or semi-explicit) since, owing to the extrapolation step 1, it allows the uncoupled solution of steps 2 and 4 , which are computational demanding. The interested reader is referred to [32, Section 4.6] for an analogous approach, using a different time discretization scheme and to 48, 58, 10, 23 for a description of various computational techniques (preconditioning, parallel computing, etc.) used for the numerical resolution of the bidomain equations.

\subsection{Partitioned heart-torso coupling}

At each time step, the linear problem 3.14 requires the coupled solution of the transmembrane potential $V_{\mathrm{m}}^{n+1}$ and the heart-torso potential $u^{n+1}$. This coupling can be solved monolithically, that is, after full assembling of the whole system matrix (see e.g. [32, Sections 4.6 and 4.5.1] and [52, 51, 8]). But this results in a increased number of unknowns with respect to the original bidomain 
system. Moreover, this procedure is less modular since the bidomain and torso equations cannot be solved independently.

This shortcoming can be overcome using a partitioned iterative procedure based on domain decomposition (see e.g. [46, 53]). In this study, the heart-torso coupling is solved using the so-called Dirichlet-Neumann algorithm, combined with a specific acceleration strategy. A related approach is adopted in [8] (see also [32, 44]), using an integral formulation of the torso equation (2.11).

The main idea consists in $(k$-)iterating between the heart and torso equations via the interface conditions

$$
\left\{\begin{aligned}
u_{\mathrm{T}}^{n+1, k+1} & =u_{\mathrm{e}}^{n+1, k}, & & \text { on } \quad \Sigma, \\
\boldsymbol{\sigma}_{\mathrm{e}} \boldsymbol{\nabla} u_{\mathrm{e}}^{n+1, k+1} \cdot \boldsymbol{n} & =\boldsymbol{\sigma}_{\mathrm{T}} \boldsymbol{\nabla} u_{\mathrm{T}}^{n+1, k+1} \cdot \boldsymbol{n}, & & \text { on } \quad \Sigma .
\end{aligned}\right.
$$

Hence, the monolithic solution is recovered at convergence. In the framework of (3.14 2 , this amounts to decompose the discrete test function space $W_{h}$ as the direct sum $W_{h}=Z_{h, 0} \oplus \mathcal{L} V_{h}$. The subspace $Z_{h, 0}$ contains the functions of $W_{h}$ vanishing in $\overline{\Omega_{\mathrm{H}}}$, whereas $\mathcal{L} V_{h}$ is the range of the standard extension operator $\mathcal{L}: V_{h} \rightarrow W_{h}$ satisfying, for all $\psi_{\mathrm{e}} \in V_{h}$,

$$
\left\{\begin{aligned}
\mathcal{L} \psi_{\mathrm{e}}=\psi_{\mathrm{e}}, & \text { in } \overline{\Omega_{\mathrm{H}}} \\
\mathcal{L} \psi_{\mathrm{e}}=0, & \text { on } \Gamma_{\text {ext }}
\end{aligned}\right.
$$

The full algorithm used in this paper to solve (3.14 reads as follows: For $k \geq 0$, until convergence,

- Torso solution (Dirichlet):

$$
\begin{aligned}
& u_{\mathrm{T}}^{n+1, k+1}=u_{\mathrm{e}}^{n+1, k}, \text { on } \Sigma, \\
& \int_{\Omega_{\mathrm{T}}} \boldsymbol{\sigma}_{\mathrm{T}} \boldsymbol{\nabla} u_{\mathrm{T}}^{n+1, k+1} \cdot \nabla \psi_{\mathrm{T}}=0, \quad \forall \psi_{\mathrm{T}} \in Z_{h, 0} .
\end{aligned}
$$

- Heart-bidomain solution (Neumann):

$$
\left\{\begin{array}{c}
A_{\mathrm{m}} \int_{\Omega_{\mathrm{H}}} \frac{C_{\mathrm{m}}}{\delta t}\left(\frac{3}{2} V_{\mathrm{m}}^{n+1, k+1}-2 V_{\mathrm{m}}^{n}+\frac{1}{2} V_{\mathrm{m}}^{n-1}\right) \phi \\
+\int_{\Omega_{\mathrm{H}}} \boldsymbol{\sigma}_{\mathrm{i}} \nabla\left(V_{\mathrm{m}}^{n+1, k+1}+\widehat{u_{\mathrm{e}}^{n+1, k+1}}\right) \cdot \nabla \phi \\
=A_{\mathrm{m}} \int_{\Omega_{\mathrm{H}}}\left(I_{\mathrm{app}}\left(t_{n+1}\right)-I_{\mathrm{ion}}\left(\widetilde{V}_{\mathrm{m}}^{n+1}, w^{n+1}\right)\right) \phi \\
\int_{\Omega_{\mathrm{H}}}\left(\boldsymbol{\sigma}_{\mathrm{i}}+\boldsymbol{\sigma}_{\mathrm{e}}\right) \nabla u_{\mathrm{e}}^{\widehat{n+1, k+1}} \cdot \nabla \psi_{\mathrm{e}}+\int_{\Omega_{\mathrm{H}}} \boldsymbol{\sigma}_{\mathrm{i}} \boldsymbol{\nabla} V_{\mathrm{m}}^{n+1, k+1} \cdot \nabla \psi_{\mathrm{e}} \\
=-\int_{\Omega_{\mathrm{T}}} \boldsymbol{\sigma}_{\mathrm{T}} \boldsymbol{\nabla} u_{\mathrm{T}}^{n+1, k+1} \cdot \nabla \mathcal{L} \psi_{\mathrm{e}},
\end{array}\right.
$$

for all $\phi \in V_{h}$ and $\psi_{\mathrm{e}} \in V_{h}$, with $\int_{\Omega_{\mathrm{H}}} \psi_{\mathrm{e}}=0$.

- Relaxation step:

$$
u_{\mathrm{e}}^{n+1, k+1}{ }_{\mid \Sigma} \longleftarrow \omega_{k} \widehat{u_{\mathrm{e}}^{n+1, k+1}}{ }_{\mid \Sigma}+\left(1-\omega_{k}\right) u_{\mathrm{e}}^{n+1, k}{ }_{\mid \Sigma} .
$$


The coefficient $\omega_{k}$ is a dynamic relaxation parameter which aims to accelerate the convergence of the iterations. In this work, the following explicit expression, based on a multidimensional Aitken formula (see e.g. [29]), has been considered

$$
\omega_{k}=\frac{\left(\lambda^{k}-\lambda^{k-1}\right) \cdot\left(\lambda^{k}-\widehat{\lambda}^{k+1}-\lambda^{k-1}+\widehat{\lambda}^{k}\right)}{\left|\lambda^{k}-\widehat{\lambda}^{k+1}-\lambda^{k-1}+\widehat{\lambda}^{k}\right|^{2}}, \quad \lambda^{k} \stackrel{\text { def }}{=} u_{\mathrm{e}}^{n+1, k}{ }_{\mid \Sigma} \cdot
$$

\section{Numerical results}

In this section, it is shown that the full PDE/ODE based model RM, completed by additional modeling assumptions, allows to get meaningful 12-lead ECG signals. Moreover, the predictive capabilities of the model are illustrated by providing realistic numerical ECG signals for some known pathologies, without any other calibration of the model than those directly related to the pathology.

\subsection{Reference simulation}

Throughout this paper, the terminology "reference simulation" (or RS) refers to the 12-lead numerical ECG signals obtained by solving the reference model RM of section 2 with the numerical method described in section 3 and the modeling assumption described in the following paragraphs. The model parameters used in the RS are summed up in Tables 13 below and, as initial data, we have taken $V_{\mathrm{m}}^{0}=V_{\min }$ and $w^{0}=1 /\left(V_{\max }-V_{\min }\right)^{2}$.

\subsubsection{Anatomical model and computational meshes}

The torso computational geometry (see Figure 2, including the lung and main bone regions, was obtained starting from the Zygote ${ }^{1}$ model - a geometric model based on actual anatomical data - using the 3-matid ${ }^{2}$ software to obtain computationally-correct surface meshes. The heart geometry is simplified, based on intersecting ellipsoids, so that the fibers orientation can be parametrized in terms of analytical functions. We refer to [49] for the details of the geometrical definition of the heart. Note that this simplified geometry only includes the ventricles. We therefore cannot simulate the P-wave of the ECG.

The 3D computational meshes of the torso and the heart are displayed in Figures 2 and 3 . They have been obtained by processing the surface meshes with the softwares Yams 21] and GHS3D [22].

\subsubsection{Heart conductivity}

Cardiac muscle is made of fibers. The electrical conductivity is higher along the fiber direction than along the cross-fiber direction. The intracellular and extracellular media are therefore anisotropic. This anisotropy is included in our model defining the conductivity tensors $\boldsymbol{\sigma}_{\mathrm{i}}$ and $\boldsymbol{\sigma}_{\mathrm{e}}$ by:

$$
\boldsymbol{\sigma}_{\mathrm{i}, \mathrm{e}}(x) \stackrel{\text { def }}{=} \sigma_{\mathrm{i}, \mathrm{e}}^{\mathrm{t}} \boldsymbol{I}+\left(\sigma_{\mathrm{i}, \mathrm{e}}^{\mathrm{l}}-\sigma_{\mathrm{i}, \mathrm{e}}^{\mathrm{t}}\right) \boldsymbol{a}(x) \otimes \boldsymbol{a}(x),
$$

\footnotetext{
1 www.3dscience.com
}

2 WwW.materialise.com 


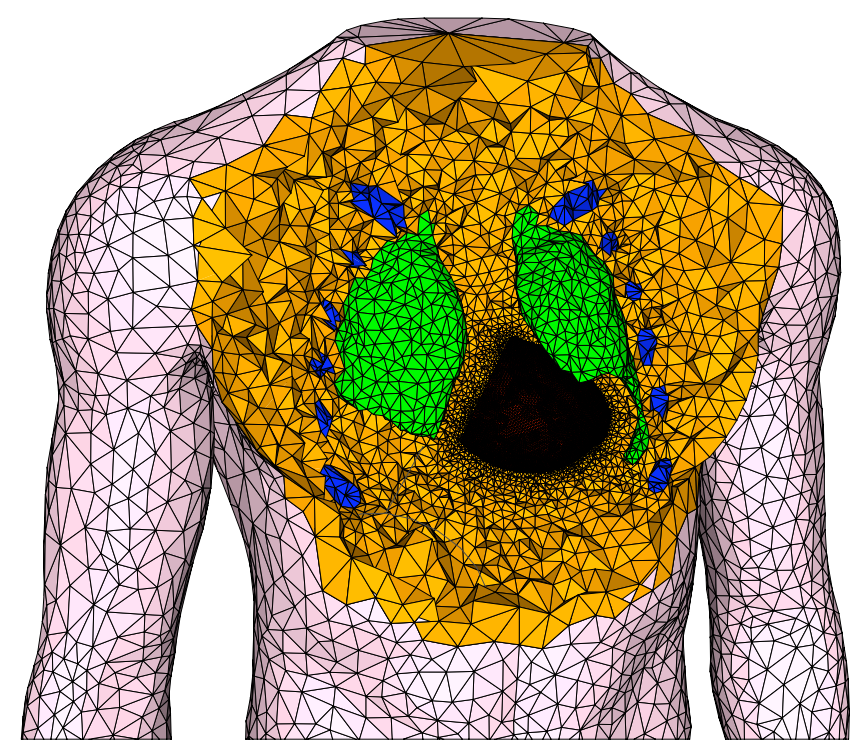

Figure 2: Computational torso mesh.
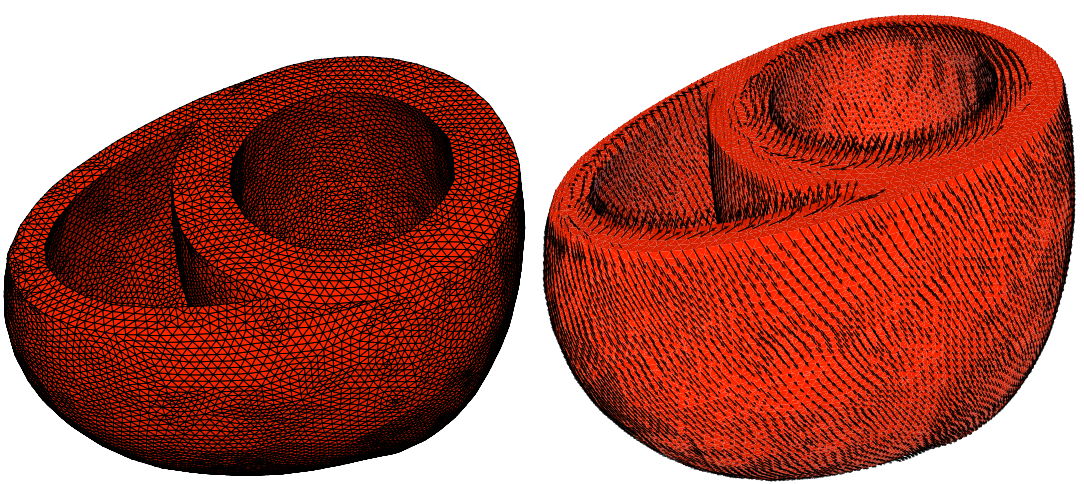

Figure 3: Computational heart mesh (left) and heart fiber directions (right).

where $\boldsymbol{a}(x)$ is a unit vector parallel to the local fiber direction (Figure 3) and $\sigma_{\mathrm{i}, e}^{\mathrm{l}}$ and $\sigma_{\mathrm{i}, e}^{\mathrm{t}}$ are respectively the conductivity coefficients in the intra- and extracellular media measured along the fibers direction and in the transverse direction. Different conductivities values are available in the literature (see e.g. [51, 9, 35]). The values used in our simulations, originally reported in [42], are given in Table 1. As mentioned above, the fibers directions have been set as in [49.

\begin{tabular}{|c|c|c|c|}
\hline $\boldsymbol{\sigma}_{\mathrm{i}}^{1}\left(\mathrm{~S} \mathrm{~cm}^{-1}\right)$ & $\boldsymbol{\sigma}_{\mathrm{e}}^{1}\left(\mathrm{~S} \mathrm{~cm}^{-1}\right)$ & $\boldsymbol{\sigma}_{\mathrm{i}}^{\mathrm{t}}\left(\mathrm{S} \mathrm{cm}^{-1}\right)$ & $\boldsymbol{\sigma}_{\mathrm{e}}^{\mathrm{t}}\left(\mathrm{S} \mathrm{cm}^{-1}\right)$ \\
\hline $3.0 \times 10^{-3}$ & $3.0 \times 10^{-3}$ & $3.0 \times 10^{-4}$ & $1.2 \times 10^{-3}$ \\
\hline
\end{tabular}

Table 1: Heart conductivity parameters. 


\subsubsection{Torso conductivity}

We assume that the torso has isotropic conductivity, i.e. $\boldsymbol{\sigma}_{\mathrm{T}}$ is diagonal $\boldsymbol{\sigma}_{\mathrm{T}}=$ $\sigma_{\mathrm{T}} \boldsymbol{I}$, and that the scalar heterogeneous conductivity $\sigma_{\mathrm{T}}$ takes three different values:

$$
\sigma_{\mathrm{T}}= \begin{cases}\sigma_{\mathrm{T}}^{\mathrm{l}}, & \text { lungs, } \\ \sigma_{\mathrm{T}}^{\mathrm{b}}, & \text { bone, } \\ \sigma_{\mathrm{T}}^{\mathrm{t}}, & \text { remaining regions, }\end{cases}
$$

given in Table 2

\begin{tabular}{|c|c|c|}
\hline$\sigma_{\mathrm{T}}^{\mathrm{l}}\left(\mathrm{S} \mathrm{cm}^{-1}\right)$ & $\sigma_{\mathrm{T}}^{\mathrm{b}}\left(\mathrm{S} \mathrm{cm}^{-1}\right)$ & $\sigma_{\mathrm{T}}^{\mathrm{t}}\left(\mathrm{S} \mathrm{cm}^{-1}\right)$ \\
\hline $2.4 \times 10^{-4}$ & $4 \times 10^{-5}$ & $6 \times 10^{-4}$ \\
\hline
\end{tabular}

Table 2: Torso conductivity parameters.

\subsubsection{His bundle and Purkinje fibers}

The His bundle quickly transmits the activation from the atrioventricular node to the ventricles. It is made of three main branches in the septum and gives rise to the thin Purkinje fibers in the ventricular muscle. The activation travels from the His bundle to the ventricular muscle in about 40ms. Interesting attempts at modelling the His bundle and the Purkinje fibers have been presented in the literature (see e.g. [57). But a physiological model of this fast conduction network coupled to a 3D model of the myocardium raises many modeling and computational difficulties: the fiber network has to be manually defined whereas it cannot be non-invasively obtained from classical imaging techniques; the results are strongly dependent on the density of fibers which is a quantity difficult to determine; the time and the space scales are quite different in the fast conduction network and in the rest of the tissue which can be challenging from the computational standpoint.

To circumvent these issues, we propose to roughly model the Purkinje system by initializing the activation with a (time-dependent) external volume current, acting on a thin subendocardial layer (both left and right parts). The propagation speed of this initial activation is a parameter of the model (see the details in appendix A. Although this approach involves a strong simplification of the reality, it allows a simple and quite accurate control of the activation initialization, which is a fundamental aspect in the simulation of correct ECGs.

\subsubsection{Cell heterogeneity}

Action potential duration (APD) heterogeneity may be found at different myocardium locations, for instance: between base and apex, between septal and posterior sides, and transmurally (see e.g. 20]). Although not yet fully explained (see e.g. [13, for a review), experimental evidence [27, 20, 59, 2] suggests that transmural APD heterogeneity is likely to be the most important factor in the genesis of the normal ECG T-wave shape and polarity. A number 
of simulation studies [14, 41, 40, 30, 6, confirm also this (still debated) postulate. Interestingly, the numerical investigations recently reported in [1] (using a highly idealized geometry) indicate that the polarity of the T-wave (for unipolar ECG leads) may be mainly driven by the cardiac tissue anisotropy.

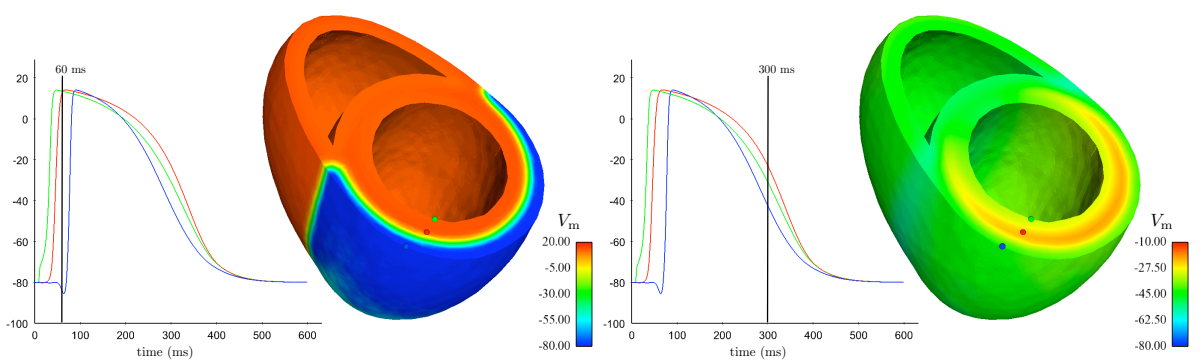

Figure 4: Transmural APD heterogeneity: comparison of the simulated transmembrane potentials for endocardial cells (green), M-cells (red) and epicardial cells (blue). Snapshots of the transmembrane potential at times $t=60$ and 300 ms.

In the present work, cell heterogeneity is only considered as transmural variation of APD in the left ventricle. Hence, we assume that epicardial cells have the shortest APD and that endocardial cells have an intermediate APD between mid-myocardial cells (M-cells) and epicardial cells (see e.g. [59]). From the analysis reported in [36, Section 3.1], the leading order of the maximum APD provided by the Mitchell-Schaeffer ionic model 2.6) is proportional to the parameter $\tau_{\text {close }}$. Thus, the APD heterogeneity is modeled with a parameter $\tau_{\text {close }}$ varying across the left ventricle transmural direction: $\tau_{\text {close }}^{\text {endo }}$ near the endocardium, $\tau_{\text {close }}^{\text {mcell }}$ in the mid-myocardium (M-cells) and $\tau_{\text {close }}^{\text {epi }}$ near the epicardium (see Figure 4). For simplicity, we take a constant value of $\tau_{\text {close }}^{\mathrm{RV}}$ in the whole right ventricle. The values of the parameters are given in Table 3 .

\begin{tabular}{|c|c|c|c|c|c|c|c|c|c|c|c|}
\hline$A_{\mathrm{m}}\left(\mathrm{cm}^{-1}\right)$ & $C_{\mathrm{m}}(\mathrm{mF})$ & $\tau_{\text {in }}$ & $\tau_{\text {out }}$ & $\tau_{\text {open }}$ & $\tau_{\text {close }}^{\text {RV }}$ & $\tau_{\text {close }}^{\text {endo }}$ & $\tau_{\text {close }}^{\text {mcell }}$ & $\tau_{\text {close }}^{\text {epi }}$ & $V_{\text {gate }}$ & $V_{\min }$ & $V_{\max }$ \\
\hline 200 & $10^{-3}$ & 4.5 & 90 & 100 & 120 & 130 & 140 & 90 & -67 & -80 & 20 \\
\hline
\end{tabular}

Table 3: Cell membrane parameters.

\subsubsection{Results}

The ECGs are computed according to the standard 12-lead ECG definition (see [35], for instance):

$$
\begin{aligned}
& \mathrm{I} \stackrel{\text { def }}{=} u_{\mathrm{T}}(L)-u_{\mathrm{T}}(R), \quad \mathrm{II} \stackrel{\text { def }}{=} u_{\mathrm{T}}(F)-u_{\mathrm{T}}(R), \quad \text { III } \stackrel{\text { def }}{=} u_{\mathrm{T}}(F)-u_{\mathrm{T}}(L), \\
& \mathrm{aVR} \stackrel{\text { def }}{=} \frac{3}{2}\left(u_{\mathrm{T}}(R)-u_{\mathrm{W}}\right), \quad \mathrm{aVL} \stackrel{\text { def }}{=} \frac{3}{2}\left(u_{\mathrm{T}}(L)-u_{\mathrm{W}}\right), \quad \mathrm{aVF} \stackrel{\text { def }}{=} \frac{3}{2}\left(u_{\mathrm{T}}(F)-u_{\mathrm{W}}\right), \\
& \mathrm{V} i \stackrel{\text { def }}{=} u_{\mathrm{T}}\left(V_{i}\right)-u_{\mathrm{W}} \quad i=1, . ., 6,
\end{aligned}
$$




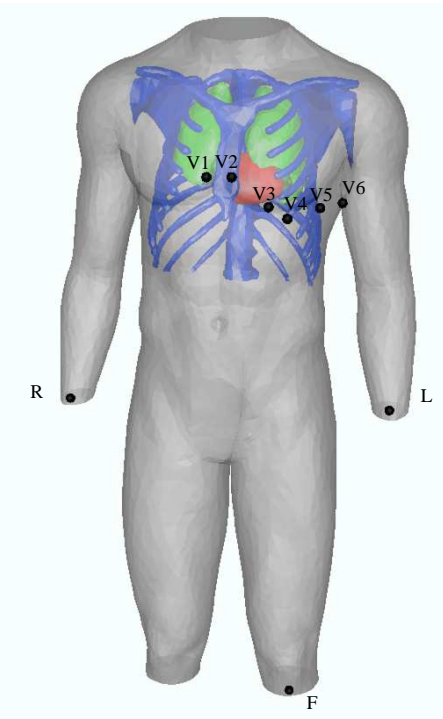

Figure 5: Torso domain: ECG leads locations.

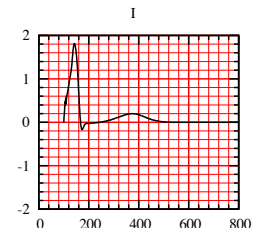

II

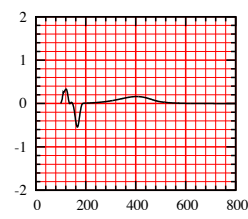

III

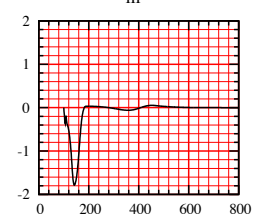

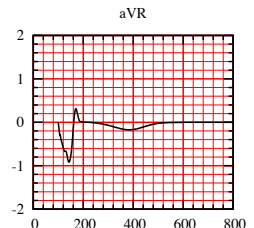

aVL

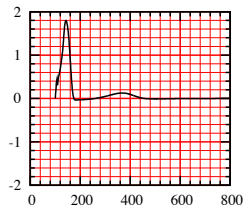

aVF

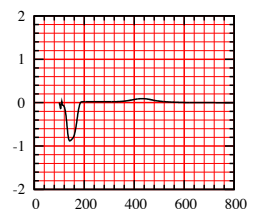

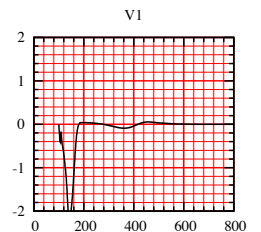

$\mathrm{v} 2$

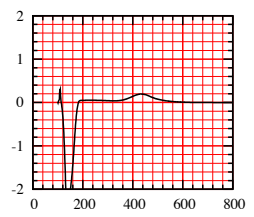

v3

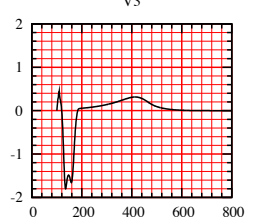

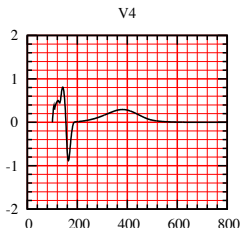

v5

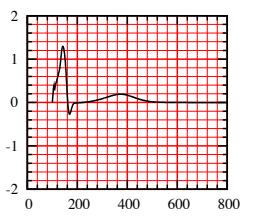

v6

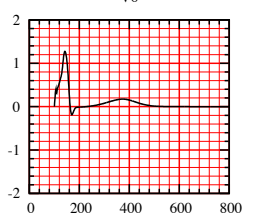

Figure 6: Reference simulation: 12-lead ECG signals obtained by a strong coupling with the torso, including anisotropy and APD heterogeneity. As usual, the units in the $x$ - and $y$-axis are $\mathrm{ms}$ and $\mathrm{mV}$, respectively.

where $u_{\mathrm{W}} \stackrel{\text { def }}{=}\left(u_{\mathrm{T}}(L)+u_{\mathrm{T}}(R)+u_{\mathrm{T}}(F)\right) / 3$ and the body surface electrode locations $L, R, F,\left\{V_{i}\right\}_{i=1, \ldots, 6}$ are indicated in Figure 5 .

The simulated ECG obtained from RS is reported in Figure 6. Some snapshots of the corresponding body surface potential are depicted in Figure 7 . Compared to a physiological ECG, the computed ECG has some minor flaws. 


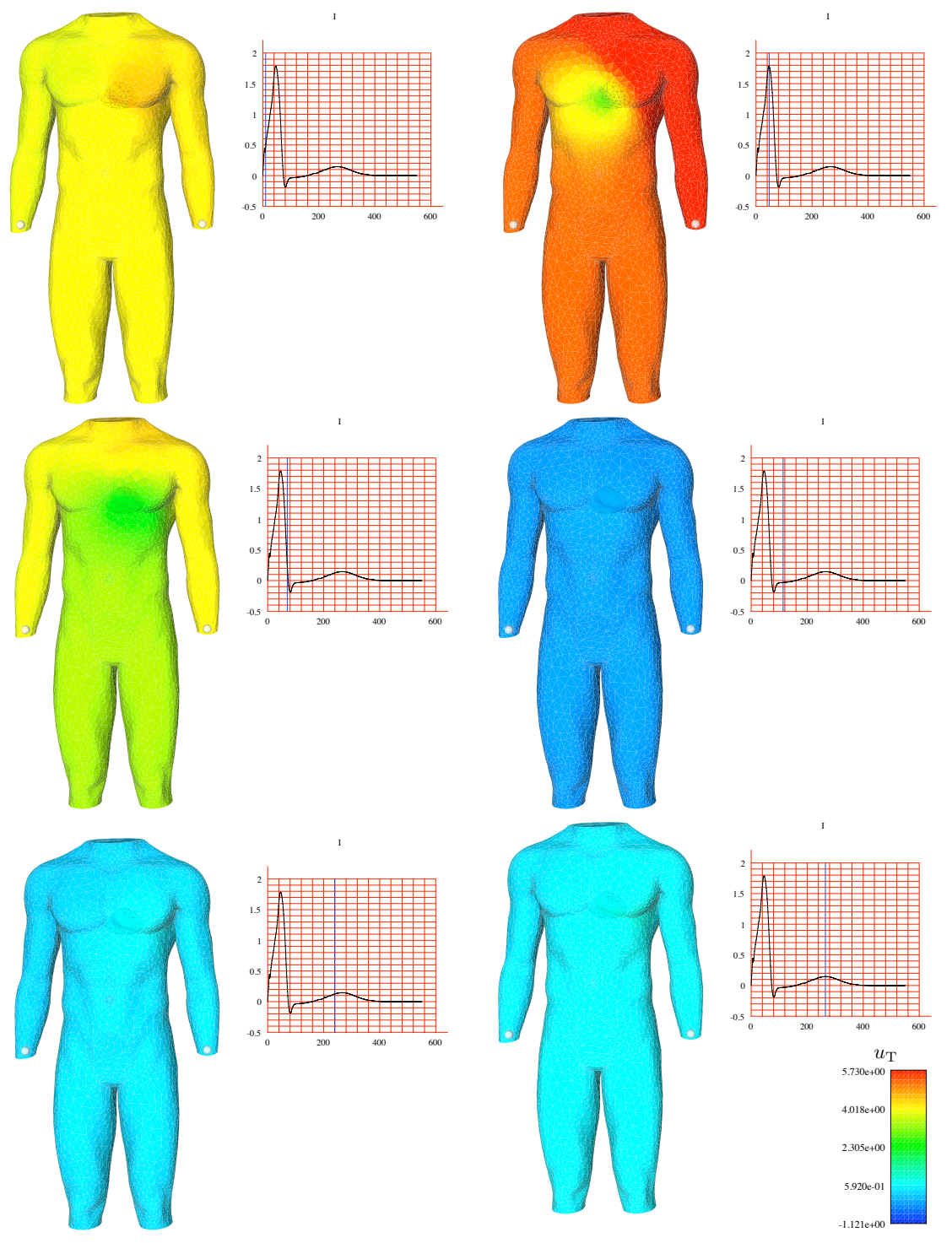

Figure 7: Reference simulation: some snapshots of the body surface potentials at times $t=10,47,70,114,239$ and $265 \mathrm{~ms}$ (from left to right and top to bottom). 
First, the T-wave amplitude is slightly lower than expected. Second, the electrical heart axis (i.e. the mean frontal plane direction of the depolarization wave traveling through the ventricles during ventricular activation) is about -40 degrees whereas it should be between 0 and 90 degrees (see e.g. [1]). This is probably due to a too horizontal position of the heart in the thoracic cavity. Third, in the precordial leads, the R-wave presents abnormal (low) amplitudes in V1 and V2 and the QRS complex shows transition from negative to positive polarity in V4 whereas this could be expected in V3.

Despite that, the main features of a physiological ECG can be observed. For example, the QRS-complex has a correct orientation and a realistic amplitude in each of the 12 leads. In particular, it is negative in lead V1 and becomes positive in lead V6. Moreover, its duration is between $80 \mathrm{~ms}$ and $120 \mathrm{~ms}$, which is the case of a healthy subject. The orientation and the duration of the T-wave are also satisfactory. To the best of our knowledge, this 12-lead ECG is the most realistic ever published from a fully based PDE/ODE 3D computational model.

\subsection{Pathological simulations}

In this paragraph, we modify the reference simulation that provided the "healthy" ECG (Figure 6) in order to simulate a right or a left bundle branch block (RBBB or LBBB). The purpose is to test whether the ECG produced by our model possesses the main characteristics that allow a medical doctor to detect these pathologies.

In the RS, the right and the left ventricle are activated simultaneously. Now, in order to simulate a LBBB (resp. a RBBB) the initial activation is blocked in the left (resp. right) ventricle.
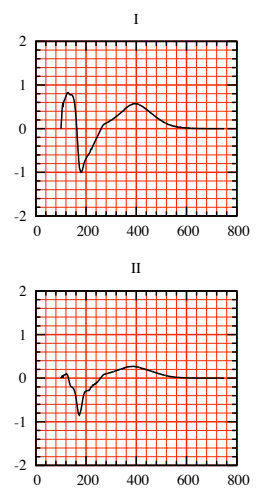

III

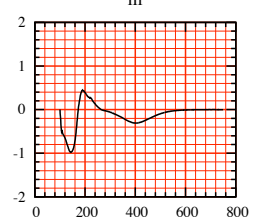

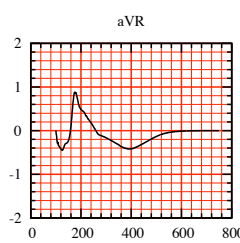

$\mathrm{aVL}$

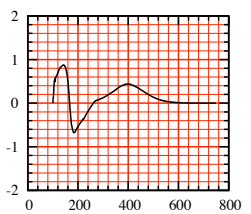

aVF

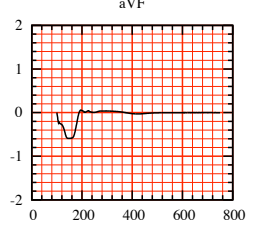

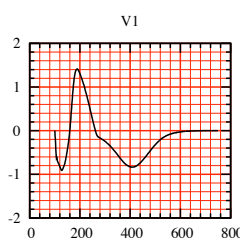

$\mathrm{V} 2$

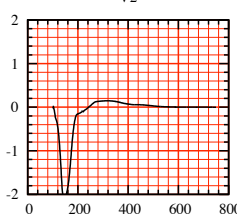

v3

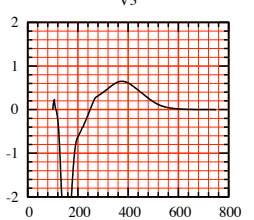

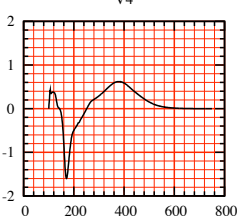

v5

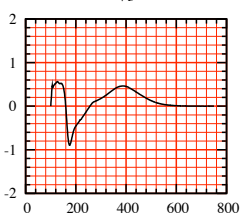

v6

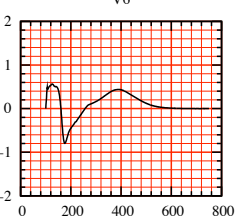

Figure 8: Simulated 12-lead ECG signals for a RBBB 

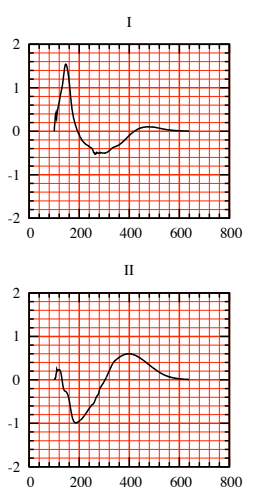

III

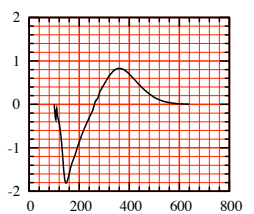

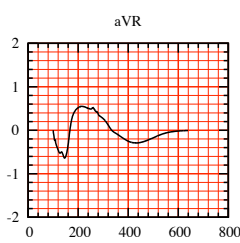

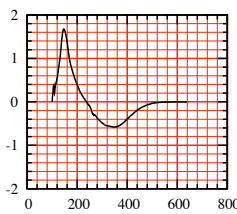

aVF

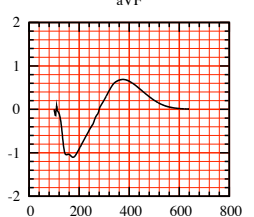

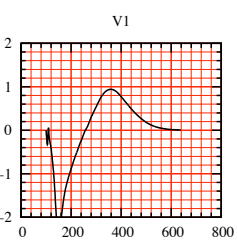

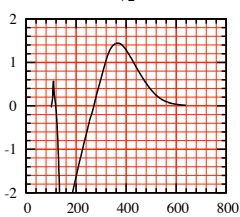

v3

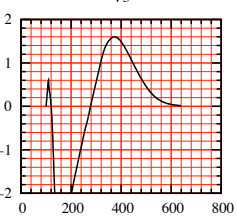

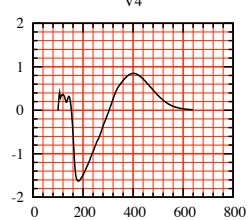

v5
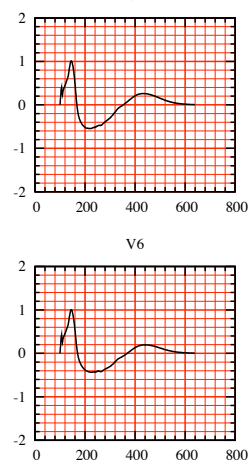

Figure 9: Simulated 12-lead ECG signals for a LBBB

The results are reported in Figure $8(\mathrm{RBBB})$ and 9 (LBBB). As in the healthy case, an expert would detect some flaws in these ECGs. For example, he would expect a larger QRS and a lead V1 without Q-wave. Nevertheless, he would also recognize the main features that indicate the bundle branch blocks (see e.g. 35]). First, the QRS-complex exceeds $120 \mathrm{~ms}$ in both cases. Second, it can be seen in Figure 8 that the duration between the beginning of the QRS complex and its last positive wave in V1 exceeds $40 \mathrm{~ms}$ which is a sign of RBBB. Third, it can be seen in Figure 9 that the duration between the beginning of the QRS complex and its last positive wave in V6 exceeds $40 \mathrm{~ms}$ which is a sign of LBBB. It is noticeable that these results have been obtained without any recalibration of the $\mathbf{R S}$, besides the above mentioned (natural) modifications needed to model the disease.

\section{$5 \quad$ Impact of some modeling assumptions}

In this section, the impact of some alternative modeling assumptions on the simulated ECG is investigated. This allows to assess to what extent the modeling assumptions involved in the $\mathbf{R S}$ are necessary to obtain a meaningful ECG.

\section{$5.1 \quad$ Heart-torso uncoupling}

A common approach to reduce the computational complexity of the $\mathbf{R M}$ consists in uncoupling the computation of $\left(V_{\mathrm{m}}, u_{\mathrm{e}}\right)$ and $u_{\mathrm{T}}$. This can be achieved by neglecting, in (2.10), the electrical torso feedback on the cardiac region. That is, by replacing the coupling condition 2.10$)_{2}$ by

$$
\boldsymbol{\sigma}_{\mathrm{e}} \boldsymbol{\nabla} u_{\mathrm{e}} \cdot \boldsymbol{n}=0, \quad \text { on } \quad \Sigma,
$$

which amounts to work with an isolated heart domain (see e.g. [9, 42]). 
As a result, the intracardiac quantities $\left(V_{\mathrm{m}}, u_{\mathrm{e}}\right)$ can be obtained, independently of $u_{\mathrm{T}}$, by solving (2.7) with initial condition (2.8) and insulating conditions

$$
\left\{\begin{array}{rll}
\boldsymbol{\sigma}_{\mathrm{i}} \nabla V_{\mathrm{m}} \cdot \boldsymbol{n}+\boldsymbol{\sigma}_{\mathrm{i}} \nabla u_{\mathrm{e}} \cdot \boldsymbol{n}=0, & \text { on } \quad \Sigma, \\
\boldsymbol{\sigma}_{\mathrm{e}} \boldsymbol{\nabla} u_{\mathrm{e}} \cdot \boldsymbol{n}=0, & \text { on } \quad \Sigma .
\end{array}\right.
$$

Thereafter, the torso potential $u_{\mathrm{T}}$ is recovered by solving (2.11) with

$$
\left\{\begin{array}{rll}
u_{\mathrm{T}}=u_{\mathrm{e}}, & \text { on } & \Sigma, \\
\boldsymbol{\sigma}_{\mathrm{T}} \boldsymbol{\nabla} u_{\mathrm{T}} \cdot \boldsymbol{n}_{\mathrm{T}}=0, & \text { on } & \Gamma_{\mathrm{ext}},
\end{array}\right.
$$

as boundary conditions. In other words, the uncoupled heart potential $u_{\mathrm{e}}$ is transferred, from $\Omega_{\mathrm{H}}$ to $\Omega_{\mathrm{T}}$, through the interface $\Sigma$ (see [3, 50]).

Remark 5.1 Rather than interface based, as (5.20), most of the uncoupled approaches reported in the literature are volume based (see [32, Section 4.2.4] for a review). Thus, the torso potentials are generated by assuming a (multi-)dipole representation of the cardiac source, typically based on the transmembrane potential gradient $\nabla V_{\mathrm{m}}$ (see e.g. [26, 44]).

From the numerical point of view, the heart-torso uncoupling amounts to replace step 4 , in section 3.1 , by:

- Solving for $\left(V_{\mathrm{m}}^{n+1}, u_{\mathrm{e}}^{n+1}\right) \in V_{h} \times V_{h}$, with $\int_{\Omega_{\mathrm{H}}} u_{\mathrm{e}}^{n+1}=0$ :

$$
\left\{\begin{array}{r}
A_{\mathrm{m}} \int_{\Omega_{\mathrm{H}}} \frac{C_{\mathrm{m}}}{\delta t}\left(\frac{3}{2} V_{\mathrm{m}}^{n+1}-2 V_{\mathrm{m}}^{n}+\frac{1}{2} V_{\mathrm{m}}^{n-1}\right) \phi+\int_{\Omega_{\mathrm{H}}} \boldsymbol{\sigma}_{\mathrm{i}} \boldsymbol{\nabla}\left(V_{\mathrm{m}}^{n+1}+u^{n+1}\right) \cdot \nabla \phi \\
=A_{\mathrm{m}} \int_{\Omega_{\mathrm{H}}}\left(I_{\mathrm{app}}\left(t_{n+1}\right)-I_{\mathrm{ion}}\left(\widetilde{V}_{\mathrm{m}}^{n+1}, w^{n+1}\right)\right) \phi \\
\int_{\Omega_{\mathrm{H}}}\left(\boldsymbol{\sigma}_{\mathrm{i}}+\boldsymbol{\sigma}_{\mathrm{e}}\right) \nabla u_{\mathrm{e}}^{n+1} \cdot \nabla \psi_{\mathrm{e}}+\int_{\Omega_{\mathrm{H}}} \boldsymbol{\sigma}_{\mathrm{i}} \nabla V_{\mathrm{m}}^{n+1} \cdot \nabla \psi_{\mathrm{e}}=0
\end{array}\right.
$$

for all $\left(\phi, \psi_{\mathrm{e}}\right) \in V_{h} \times V_{h}$, with $\int_{\Omega_{\mathrm{H}}} \psi_{\mathrm{e}}=0$.

Then, once $\left\{u_{\mathrm{e}}^{n+1}\right\}_{0 \leq n \leq N-1}$ are available, the torso potential is obtained by solving, for $u_{\mathrm{T}}^{n+1} \in \bar{Z}_{h}$,

$$
\begin{gathered}
u_{\mathrm{T}}^{n+1}=u_{\mathrm{e}}^{n+1}, \quad \text { on } \Sigma, \\
\int_{\Omega_{\mathrm{T}}} \boldsymbol{\sigma}_{\mathrm{T}} \boldsymbol{\nabla} u_{\mathrm{T}}^{n+1} \cdot \nabla \psi_{\mathrm{T}}=0, \quad \forall \psi_{\mathrm{T}} \in Z_{h, 0} .
\end{gathered}
$$

The remainder of this section discusses the impact of the uncoupled approach on ECG accuracy and computational cost.

\subsubsection{Numerical results}

Figure 10 presents the ECGs obtained with the fully coupled (i.e. the RS) and the uncoupled approaches in a healthy condition. For the sake of conciseness, we have only reported the I, aVR, V1 and V4 leads of the ECG. Figure 11 reports the comparison in the case of a pathological RBBB situation. 

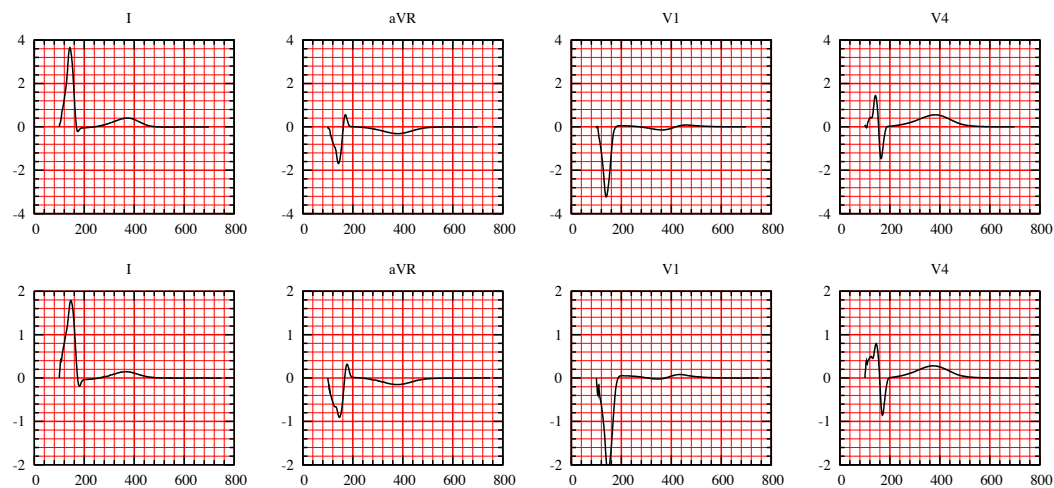

$\mathrm{aVR}$

$\mathrm{V} 1$
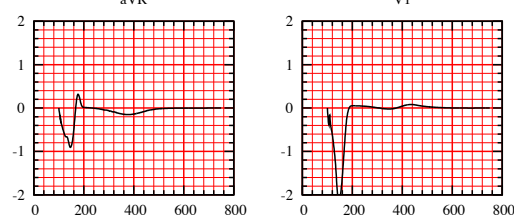

$\mathrm{v} 4$

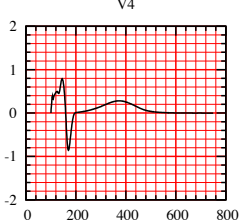

Figure 10: Comparison of the simulated healthy ECGs obtained using hearttorso uncoupling (top) and fully heart-torso coupling (bottom).

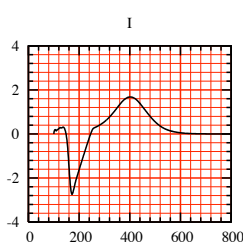

I

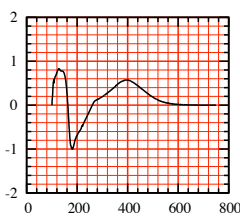

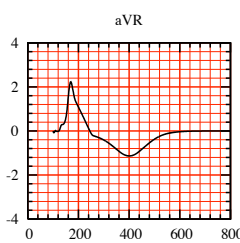

aVR

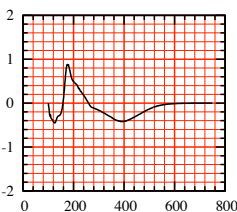

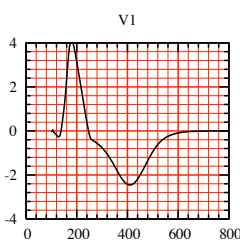

$\mathrm{V} 1$

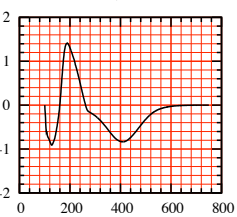

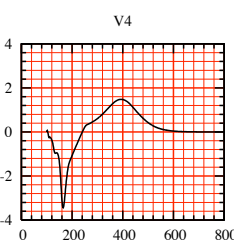

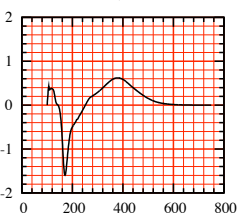

Figure 11: Comparison of the simulated RBBB ECGs obtained using heart-torso uncoupling (top) and fully heart-torso coupling (bottom).

In both cases, the amplitude of the waves of the uncoupled formulation is much larger than in the fully coupled formulation. In the healthy case (Figure 10), it can nevertheless be noted that the shape of the ECG is almost unaffected. These results are consistent with the experimental findings reported in 25]: no significant changes in epicardial activation but substantial increasing in epicardial potentials magnitude were observed when the heart surface was exposed to insulating air. Thus, considering an uncoupled formulation can be reasonable to get a qualitatively correct ECGs, in the sense that some important features of the ECGs - for example, the QRS or the QT intervals - are the same as in the fully coupled case. This observation is the basis of the numerical study reported in section 6 using heart-torso uncoupling. Nevertheless, Figure 11 shows that both amplitude and shape can differ in some cases. The uncoupling assumption has therefore to be considered with caution. Similar conclusions are given in [44, Page 315] (see also [32, Section 4.3]), by comparing the surface potentials, on a $2 \mathrm{D}$ torso slice, obtained with a multi-dipole representation of the cardiac source (see remark 5.1). 


\subsubsection{Torso transfer matrix computation}

Under a heart-torso uncoupling assumption, the torso potential $u_{\mathrm{T}}$ is computed by solving the generalized Laplace equation 2.11 with boundary conditions (5.20). Therefore, $u_{\mathrm{T}}$ depends linearly on the heart extracellular potential at the heart-torso interface $u_{\mathrm{e}}{ }_{\Sigma}$. At the discrete level, we will see that this leads to a matrix-vector product representation of the ECG computation in terms of the discrete extracellular potential at the heart-torso interface $\Sigma$.

To this aim, we introduce some additional notation and assume that the heart and torso finite element discretizations match at the interface. For the sake of simplicity, the degrees of freedom (DOF) of torso potential are partitioned as $\boldsymbol{x}_{\mathrm{T}} \stackrel{\text { def }}{=}\left[\boldsymbol{x}_{\mathrm{T}, \mathrm{I}}, \boldsymbol{x}_{\mathrm{T}, \Sigma}\right] \in \mathbb{R}^{n_{\mathrm{I}}+n_{\Sigma}}$, where $\boldsymbol{x}_{\mathrm{T}, \Sigma}$ denotes the heart-torso interface DOF and $\boldsymbol{x}_{\mathrm{T}, \mathrm{I}}$ the remaining DOF. We denote by $\boldsymbol{x}_{\mathrm{e} \mid \Sigma} \in \mathbb{R}^{n_{\Sigma}}$ the extracellular potential DOF at the heart-torso interface $\Sigma$. Finally, we assume that the 9 potential values generating the ECG (see section 4.1.6), say $\boldsymbol{x}_{\mathrm{ECG}} \in \mathbb{R}^{9}$, are obtained from the discrete torso potential $\boldsymbol{x}_{\mathrm{T}}$ in terms of an interpolation operator $\boldsymbol{P} \in \mathbb{R}^{9 \times n_{\mathrm{I}}}$, so that

$$
\boldsymbol{x}_{\mathrm{ECG}}=\boldsymbol{P} \boldsymbol{x}_{\mathrm{T}, \mathrm{I}},
$$

for instance, $\boldsymbol{P}$ can be a nodal value extraction of $\boldsymbol{x}_{\mathrm{T}, \mathrm{I}}$. On the other hand, from 5.21, the discrete torso potential $\boldsymbol{x}_{\mathrm{T}}$ is solution to the following finite element linear system:

$$
\left[\begin{array}{cc}
\boldsymbol{A}_{\mathrm{II}} & \boldsymbol{A}_{\mathrm{I} \Sigma} \\
\mathbf{0} & \boldsymbol{I}_{\Sigma \Sigma}
\end{array}\right] \quad\left[\begin{array}{c}
\boldsymbol{x}_{\mathrm{T}, \mathrm{I}} \\
\boldsymbol{x}_{\mathrm{T}, \Sigma}
\end{array}\right]=\left[\begin{array}{c}
\mathbf{0} \\
\boldsymbol{x}_{\mathrm{e} \mid \Sigma}
\end{array}\right]
$$

Hence, by Gaussian elimination, we have that $\boldsymbol{x}_{\mathrm{T}, \mathrm{I}}=-\boldsymbol{A}_{\mathrm{II}}^{-1} \boldsymbol{A}_{\mathrm{I} \Sigma} \boldsymbol{x}_{\mathrm{e} \mid \Sigma}$, and by inserting this expression in 5.22 , we obtain

$$
\boldsymbol{x}_{\mathrm{ECG}}=\underbrace{-\boldsymbol{P} \boldsymbol{A}_{\mathrm{II}}^{-1} \boldsymbol{A}_{\mathrm{I} \Sigma}}_{\boldsymbol{T}} \boldsymbol{x}_{\mathrm{e} \mid \Sigma} .
$$

Therefore, the ECG can be computed from the discrete extracellular potential at the heart torso interface, $\boldsymbol{x}_{\mathrm{e} \mid \Sigma}$, by a simple matrix-vector operation $\boldsymbol{x}_{\mathrm{ECG}}=$ $\boldsymbol{T} \boldsymbol{x}_{\mathrm{e} \mid \Sigma}$, with $\boldsymbol{T} \stackrel{\text { def }}{=}-\boldsymbol{P} \boldsymbol{A}_{\mathrm{II}}^{-1} \boldsymbol{A}_{\mathrm{I} \Sigma}$.

There are different solutions to compute $\boldsymbol{T}$. The naive idea consisting of computing the matrix $\boldsymbol{A}_{\mathrm{II}}^{-1}$ is of course ruled out. A reasonable and natural option is to compute matrix $\boldsymbol{T}$ by column (see 50]), i.e. by evaluating $\boldsymbol{T} \boldsymbol{e}_{i}$ for $i=1, \ldots, n_{\Sigma}$, where $\boldsymbol{e}_{i}$ denotes the $i$-th canonical vector of $\mathbb{R}^{n_{\Sigma}}$. But each of these evaluations involve the solution of system $(5.23)$ with $\boldsymbol{x}_{\mathrm{e} \mid \Sigma}=\boldsymbol{e}_{i}$, and therefore the overall computational cost is proportional to $n_{\Sigma}$, which can be rather expensive (remember that $n_{\Sigma}$ is the number of nodes on the heart-torso interface, and is therefore of the order of several thousands). In contrast, a computation by row is much more efficient since it is only needeed to evaluate $\boldsymbol{T}^{T} \boldsymbol{e}_{i}$ for $i=1, \ldots, 9$, where $\boldsymbol{e}_{i}$ stands for the $i$-th canonical vector of $\mathbb{R}^{9}$. From the symmetry of the finite element matrix,

$$
\boldsymbol{T}^{T}=-\boldsymbol{A}_{\mathrm{I} \Sigma}^{T} \boldsymbol{A}_{\mathrm{II}}^{-T} \boldsymbol{P}^{T}=-\boldsymbol{A}_{\Sigma \mathrm{I}} \boldsymbol{A}_{\mathrm{II}}^{-1} \boldsymbol{P}^{T} .
$$

Therefore, the matrix-vector product evaluation

$$
\boldsymbol{T}^{T} \boldsymbol{e}_{i}=-\boldsymbol{A}_{\Sigma \mathrm{I}} \underbrace{\boldsymbol{A}_{\mathrm{II}}^{-1} \boldsymbol{P}^{T} \boldsymbol{e}_{i}}_{\boldsymbol{x}_{\mathrm{T}, \mathrm{I}}},
$$


can be performed in two steps as follows. First, solve for $\left[\boldsymbol{x}_{\mathrm{T}, \mathrm{I}}, \boldsymbol{x}_{\mathrm{T}, \Sigma}\right]$ the discrete source problem (depending on the linear operator $\boldsymbol{P}$ ), with homogeneous Dirichlet boundary condition on $\Sigma$ :

$$
\left[\begin{array}{cc}
\boldsymbol{A}_{\mathrm{II}} & \boldsymbol{A}_{\mathrm{I} \Sigma} \\
\mathbf{0} & \boldsymbol{I}_{\Sigma \Sigma}
\end{array}\right] \quad\left[\begin{array}{c}
\boldsymbol{x}_{\mathrm{T}, \mathrm{I}} \\
\boldsymbol{x}_{\mathrm{T}, \Sigma}
\end{array}\right]=\left[\begin{array}{c}
\boldsymbol{P}^{t} \boldsymbol{e}_{i} \\
\mathbf{0}
\end{array}\right],
$$

Second, from (5.24), evaluate the interface residual

$$
\boldsymbol{T}^{T} \boldsymbol{e}_{i}=-\boldsymbol{A}_{\Sigma \mathrm{I}} \boldsymbol{x}_{\mathrm{T}, \mathrm{I}}=-\left[\begin{array}{ll}
\boldsymbol{A}_{\Sigma \mathrm{I}} & \boldsymbol{A}_{\Sigma \Sigma}
\end{array}\right]\left[\begin{array}{c}
\boldsymbol{x}_{\mathrm{T}, \mathrm{I}} \\
\boldsymbol{x}_{\mathrm{T}, \Sigma}
\end{array}\right] .
$$

Note that, $\boldsymbol{T}^{T} \boldsymbol{e}_{i}$ is nothing but the discrete current flux through the heart-torso interface $\Sigma$, associated to the homogeneous Dirichlet condition in (5.25).

In this paper, all the numerical ECGs based on the uncoupling conditions (5.19)-(5.20) have been obtained using the matrix $\boldsymbol{T}$ presented in this paragraph (and this matrix has been computed by row).

Remark 5.2 If the operator $\boldsymbol{P}$ is a simple extraction of nodal values from the torso potential DOF, $\boldsymbol{x}_{\mathrm{T}}$, each evaluation $\boldsymbol{T}^{T} \boldsymbol{e}_{i}$, for $i=1, \ldots, 9$, can be (formally) interpreted at the continuous level as a current flux evaluation at $\Sigma$ of the problem

$$
\left\{\begin{aligned}
\operatorname{div}\left(\boldsymbol{\sigma}_{\mathrm{T}} \boldsymbol{\nabla} v\right)=\delta_{\boldsymbol{x}_{i}}, & \text { in } \quad \Omega_{\mathrm{T}}, \\
v=0, & \text { on } \quad \Sigma, \\
\boldsymbol{\sigma}_{\mathrm{T}} \boldsymbol{\nabla} v \cdot \boldsymbol{n}_{\mathrm{T}}=0, & \text { on } \quad \Gamma_{\mathrm{ext}},
\end{aligned}\right.
$$

with $\delta_{\boldsymbol{x}_{i}}$ the Dirac's delta function at the $i$-th point, $\boldsymbol{x}_{i}$, of torso potential recording on $\Gamma_{\text {ext }}$.

Remark 5.3 Note that the transfer matrix $\boldsymbol{T}$ can be computed "off-line", since it depends neither on time nor on solution in the heart. Nevertheless, this matrix has to be recomputed when the torso conductivities are modified or when dealing with dynamic torso meshes.

\begin{tabular}{|c|c|c|}
\hline Full coupling & $\begin{array}{c}\text { Uncoupling } \\
\text { Laplace equation }\end{array}$ & $\begin{array}{c}\text { Uncoupling } \\
\text { Transfer matrix }\end{array}$ \\
\hline 60 & 4 & 1 \\
\hline
\end{tabular}

Table 4: Comparison of the elapsed CPU time (dimensionless) for the computation of the ECG.

Table 4 reports the elapsed CPU time needed to simulate an ECG with three different approaches. As expected, the uncoupling assumption significantly reduces the computational cost of the ECG simulation, especially if the transfer matrix method is used to recover the torso potentials. Let us emphasize that, the last two columns of Table 4 refer to the same problem (uncoupled formulation) solved with two different algorithms, whereas the problem corresponding to the first column (fully coupled formulation) is different and a priori more accurate. 


\subsection{Study of the monodomain model}

In the previous section we have investigated a simplifying modeling assumption that allows a uncoupled computation of the heart and torso potentials $\left(V_{\mathrm{m}}, u_{\mathrm{e}}\right)$ and $u_{\mathrm{T}}$. We now discuss another simplification known as monodomain approximation (see e.g. 9, 12 ). Combined with a heart-torso uncoupling assumption, this approach leads to a fully decoupled computation of $V_{\mathrm{m}}, u_{\mathrm{e}}$ and $u_{\mathrm{T}}$.

The next subsection investigates the implications, on ECG modeling, of the general monodomain derivation proposed in [9, 12, without any assumptions on the anisotropy ratio of the intra- and extracellular conductivities. The impact of this approximation on the simulated ECG is then illustrated in subsection 5.2 .2 , using the heart-torso uncoupling simplification.

\subsubsection{The monodomain approximation}

We assume that the intra- and extracellular local conductivities $\sigma_{\mathrm{i}}^{\mathrm{l}, \mathrm{t}}$ and $\sigma_{\mathrm{e}}^{\mathrm{l}, \mathrm{t}}$ are homogeneous (constant in space). Let $\boldsymbol{j} \stackrel{\text { def }}{=} \boldsymbol{j}_{\mathrm{i}}+\boldsymbol{j}_{\mathrm{e}}$ be the total current, flowing into $\Omega_{\mathrm{H}}$, and $\boldsymbol{\sigma} \stackrel{\text { def }}{=} \boldsymbol{\sigma}_{\mathrm{i}}+\boldsymbol{\sigma}_{\mathrm{e}}$ be the bulk conductivity tensor of the medium.

From (2.3) and 2.4 , $\boldsymbol{j}=-\boldsymbol{\sigma}_{\mathrm{i}} \boldsymbol{\nabla} u_{\mathrm{i}}-\boldsymbol{\sigma}_{\mathrm{e}} \boldsymbol{\nabla} u_{\mathrm{e}}=-\boldsymbol{\sigma}_{\mathrm{i}} \boldsymbol{\nabla} V_{\mathrm{m}}-\boldsymbol{\sigma} \boldsymbol{\nabla} u_{\mathrm{e}}$, or, equivalently,

$$
\nabla u_{\mathrm{e}}=-\boldsymbol{\sigma}^{-1} \boldsymbol{\sigma}_{\mathrm{i}} \nabla V_{\mathrm{m}}-\boldsymbol{\sigma}^{-1} \boldsymbol{j} .
$$

By inserting this expression in 2.7$)_{1}$ and 2.9 , we obtain

$$
\left\{\begin{aligned}
A_{\mathrm{m}}\left(C_{\mathrm{m}} \frac{\partial V_{\mathrm{m}}}{\partial t}+I_{\mathrm{ion}}\left(V_{\mathrm{m}}, w\right)\right) & -\operatorname{div}\left(\boldsymbol{\sigma}_{\mathrm{i}}\left(\boldsymbol{I}-\boldsymbol{\sigma}^{-1} \boldsymbol{\sigma}_{\mathrm{i}}\right) \nabla V_{\mathrm{m}}\right) \\
& =-\operatorname{div}\left(\boldsymbol{\sigma}_{\mathrm{i}} \boldsymbol{\sigma}^{-1} \boldsymbol{j}\right)+A_{\mathrm{m}} I_{\mathrm{app}}, \quad \text { in } \quad \Omega_{\mathrm{H}}, \\
\boldsymbol{\sigma}_{\mathrm{i}}\left(\boldsymbol{I}-\boldsymbol{\sigma}^{-1} \boldsymbol{\sigma}_{\mathrm{i}}\right) \nabla V_{\mathrm{m}} \cdot \boldsymbol{n} & =\boldsymbol{\sigma}_{\mathrm{i}} \boldsymbol{\sigma}^{-1} \boldsymbol{j} \cdot \boldsymbol{n}, \text { on } \Sigma .
\end{aligned}\right.
$$

On the other hand, $\boldsymbol{\sigma}_{\mathrm{i}}\left(\boldsymbol{I}-\boldsymbol{\sigma}^{-1} \boldsymbol{\sigma}_{\mathrm{i}}\right)=\boldsymbol{\sigma}_{\mathrm{i}} \boldsymbol{\sigma}^{-1}\left(\boldsymbol{\sigma}-\boldsymbol{\sigma}_{\mathrm{i}}\right)=\boldsymbol{\sigma}_{\mathrm{i}} \boldsymbol{\sigma}^{-1} \boldsymbol{\sigma}_{\mathrm{e}}$. Therefore, by defining

$$
\sigma_{\mathrm{a}} \stackrel{\text { def }}{=} \sigma_{\mathrm{i}} \sigma^{-1} \sigma_{\mathrm{e}}
$$

the expression 5.27 reduces to

$$
\left\{\begin{aligned}
A_{\mathrm{m}}\left(C_{\mathrm{m}} \frac{\partial V_{\mathrm{m}}}{\partial t}+I_{\mathrm{ion}}\left(V_{\mathrm{m}}, w\right)\right) & -\operatorname{div}\left(\boldsymbol{\sigma}_{\mathrm{a}} \boldsymbol{\nabla} V_{\mathrm{m}}\right) \\
& =-\operatorname{div}\left(\boldsymbol{\sigma}_{\mathrm{i}} \boldsymbol{\sigma}^{-1} \boldsymbol{j}\right)+A_{\mathrm{m}} I_{\mathrm{app}}, \quad \text { in } \quad \Omega_{\mathrm{H}}, \\
\boldsymbol{\sigma}_{\mathrm{a}} \boldsymbol{\nabla} V_{\mathrm{m}} \cdot \boldsymbol{n} & =\boldsymbol{\sigma}_{\mathrm{i}} \boldsymbol{\sigma}^{-1} \boldsymbol{j} \cdot \boldsymbol{n}, \quad \text { on } \Sigma .
\end{aligned}\right.
$$

Following [9, 12, we deduce from 4.16

$$
\boldsymbol{\sigma}_{\mathrm{i}} \boldsymbol{\sigma}^{-1}=\mu_{\mathrm{t}} \boldsymbol{I}+\left(\mu_{\mathrm{l}}-\mu_{\mathrm{t}}\right) \boldsymbol{a} \otimes \boldsymbol{a},
$$

with

$$
\mu_{1} \stackrel{\text { def }}{=} \frac{\sigma_{1}^{\mathrm{i}}}{\sigma_{1}^{\mathrm{i}}+\sigma_{\mathrm{l}}^{\mathrm{e}}}, \quad \mu_{\mathrm{t}} \stackrel{\text { def }}{=} \frac{\sigma_{\mathrm{t}}^{\mathrm{i}}}{\sigma_{\mathrm{t}}^{\mathrm{i}}+\sigma_{\mathrm{t}}^{\mathrm{e}}},
$$

By setting $\epsilon \stackrel{\text { def }}{=}\left|\mu_{\mathrm{t}}-\mu_{\mathrm{l}}\right|$, we deduce from 5.30

$$
\boldsymbol{\sigma}_{\mathrm{i}} \boldsymbol{\sigma}^{-1}=\mu_{\mathrm{t}} \boldsymbol{I}+O(\epsilon) .
$$


As noticed in [9], $\epsilon$ is a parameter that measures the gap between the anisotropy ratios of the intra- and extracellular media. In general $0 \leq \epsilon<1$, and for equal anisotropy ratios $\epsilon=0$ so that $\boldsymbol{\sigma}_{\mathrm{i}} \boldsymbol{\sigma}^{-1}=\mu_{\mathrm{t}} \boldsymbol{I}$.

Assuming $\epsilon \ll 1$, the expansion (5.31) can be inserted into 5.29 by keeping the terms up to the zero order. Thus, since $\mu_{\mathrm{t}}$ is assumed to be constant, and using (2.1) and (2.9), up to the zero order in $\epsilon$, the so-called monodomain approximation is obtained:

$$
\left\{\begin{array}{r}
A_{\mathrm{m}}\left(C_{\mathrm{m}} \frac{\partial V_{\mathrm{m}}}{\partial t}+I_{\mathrm{ion}}\left(V_{\mathrm{m}}, w\right)\right)-\operatorname{div}\left(\boldsymbol{\sigma}_{\mathrm{a}} \boldsymbol{\nabla} V_{\mathrm{m}}\right)=A_{\mathrm{m}} I_{\mathrm{app}}, \quad \text { in } \quad \Omega_{\mathrm{H}}, \\
\boldsymbol{\sigma}_{\mathrm{a}} \boldsymbol{\nabla} V_{\mathrm{m}} \cdot \boldsymbol{n}=-\mu_{\mathrm{t}} \boldsymbol{\sigma}_{\mathrm{e}} \boldsymbol{\nabla} u_{\mathrm{e}} \cdot \boldsymbol{n}, \quad \text { on } \Sigma .
\end{array}\right.
$$

Heart-torso full coupling. Under the full coupling conditions $(2.10), V_{\mathrm{m}}$ and $u_{\mathrm{e}}$ cannot be determined independently from each other. Note that, in 5.32 the coupling between $V_{\mathrm{m}}$ and $u_{\mathrm{e}}$ is fully concentrated on $\Sigma$, whereas in RM this coupling is also distributed in $\Omega_{\mathrm{H}}$, through 2.7$]_{1}$. Therefore, as soon as the heart and the torso are strongly coupled, the monodomain approximation does not substantially reduce the computational complexity with respect to RM. Owing to this observation, we will not pursue the investigations on this approach.

Heart-torso uncoupling. Within the framework of section 5.1, the insulating condition (5.18) combined with $(5.32$ ) yields

$$
\left\{\begin{array}{r}
A_{\mathrm{m}}\left(C_{\mathrm{m}} \frac{\partial V_{\mathrm{m}}}{\partial t}+I_{\mathrm{ion}}\left(V_{\mathrm{m}}, w\right)\right)-\operatorname{div}\left(\boldsymbol{\sigma}_{\mathrm{a}} \boldsymbol{\nabla} V_{\mathrm{m}}\right)=A_{\mathrm{m}} I_{\mathrm{app}}, \quad \text { in } \quad \Omega_{\mathrm{H}}, \\
\boldsymbol{\sigma}_{\mathrm{a}} \boldsymbol{\nabla} V_{\mathrm{m}} \cdot \boldsymbol{n}=0, \quad \text { on } \quad \Sigma,
\end{array}\right.
$$

which, along with 2.5 , allows to compute $V_{\mathrm{m}}$ independently of $u_{\mathrm{e}}$. The extracellular potential can then be recovered, a posteriori, by solving

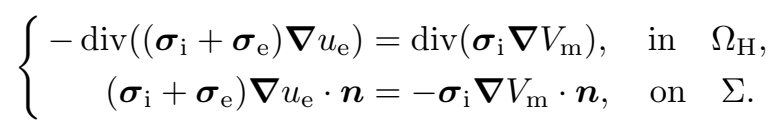

At last, the heart potentials are transferred to the torso by solving 2.11) with 5.20 , as in section 5.1 .

Therefore, the monodomain approximation 5.32 combined with a hearttorso uncoupling assumption leads to a fully decoupled computation of $V_{\mathrm{m}}, u_{\mathrm{e}}$ and $u_{\mathrm{T}}$. The three systems of equations which have to be solved successively read:

1. Monodomain problem, decoupled $V_{\mathrm{m}}$ :

$$
\left\{\begin{array}{r}
A_{\mathrm{m}}\left(C_{\mathrm{m}} \frac{\partial V_{\mathrm{m}}}{\partial t}+I_{\mathrm{ion}}\left(V_{\mathrm{m}}, w\right)\right)-\operatorname{div}\left(\boldsymbol{\sigma}_{\mathrm{a}} \boldsymbol{\nabla} V_{\mathrm{m}}\right)=A_{\mathrm{m}} I_{\mathrm{app}}, \quad \text { in } \Omega_{\mathrm{H}} \\
\frac{\partial w}{\partial t}+g\left(V_{\mathrm{m}}, w\right)=0, \quad \text { in } \Omega_{\mathrm{H}} \\
\boldsymbol{\sigma}_{\mathrm{a}} \boldsymbol{\nabla} V_{\mathrm{m}} \cdot \boldsymbol{n}=0, \quad \text { on } \Sigma .
\end{array}\right.
$$


2. Heart extracellular potential $u_{\mathrm{e}}$ :

$$
\left\{\begin{aligned}
\operatorname{div}\left(\left(\boldsymbol{\sigma}_{\mathrm{i}}+\boldsymbol{\sigma}_{\mathrm{e}}\right) \boldsymbol{\nabla} u_{\mathrm{e}}\right)=-\operatorname{div}\left(\boldsymbol{\sigma}_{\mathrm{i}} \boldsymbol{\nabla} V_{\mathrm{m}}\right), & \text { in } \quad \Omega_{\mathrm{H}}, \\
\left(\boldsymbol{\sigma}_{\mathrm{i}}+\boldsymbol{\sigma}_{\mathrm{e}}\right) \boldsymbol{\nabla} u_{\mathrm{e}} \cdot \boldsymbol{n}=-\boldsymbol{\sigma}_{\mathrm{i}} \boldsymbol{\nabla} V_{\mathrm{m}} \cdot \boldsymbol{n}, & \text { on } \Sigma .
\end{aligned}\right.
$$

3. Torso potential $u_{\mathrm{T}}$ :

$$
\left\{\begin{array}{rll}
\operatorname{div}\left(\boldsymbol{\sigma}_{\mathrm{T}} \boldsymbol{\nabla} u_{\mathrm{T}}\right)=0, & \text { in } & \Omega_{\mathrm{T}}, \\
u_{\mathrm{T}}=u_{\mathrm{e}}, & \text { on } & \Sigma, \\
\boldsymbol{\sigma}_{\mathrm{T}} \boldsymbol{\nabla} u_{\mathrm{T}} \cdot \boldsymbol{n}_{\mathrm{T}}=0, & \text { on } & \Gamma_{\text {ext }} .
\end{array}\right.
$$

To sum up the discussion of this subsection on can say that two levels of simplification can be considered with respect to $\mathbf{R M}$ : first, replacing the bidomain equations by the monodomain equations; second, replacing the full heart-torso coupling by an uncoupled formulation. The first simplification significantly reduces the computational effort only if the second one is also assumed.

\subsubsection{Numerical results with heart-torso uncoupling}

Figure 12 shows the ECG signals obtained with the bidomain model (bottom) and the monodomain approximation (top) in a healthy case, using the hearttorso uncoupling simplification. The simulated ECGs for a RBBB pathological condition are given in Figure 13. These figures clearly show that the most important clinical characteristics (e.g. QRS or QT durations) are essentially the same in both approaches.

The first lead, in a healthy case, of both approaches are presented together in Figure 14, for better comparison. The relative difference on the first lead is only $4 \%$ in $l^{2}$-norm. Thus, as far as the ECG is concerned, bidomain equations can be safely replaced by the monodomain approximation.
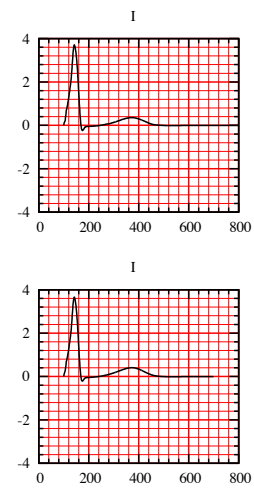

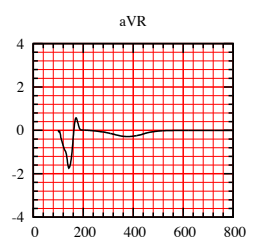

aVR

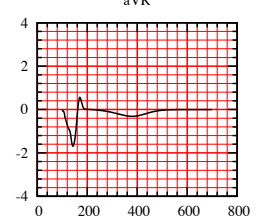

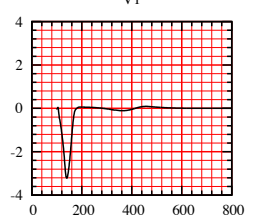

v1

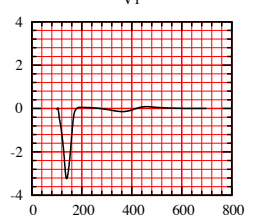

$\mathrm{v} 4$
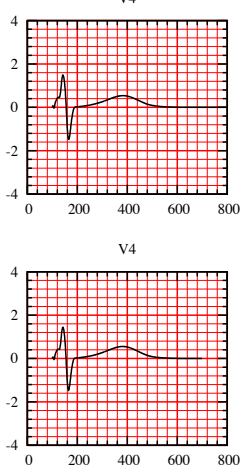

Figure 12: Simulated normal ECG with heart-torso uncoupling: monodomain (top) and bidomain (bottom) models.

These observations are consistent with the conclusions of other studies based on isolated whole heart models [9, 42. For instance, the numerical results reported in 42 show that the propagation of the activation wave is only $2 \%$ faster in the bidomain model and that the electrograms (point-wise values of the extra-cellular potential) are almost indistinguishable. 

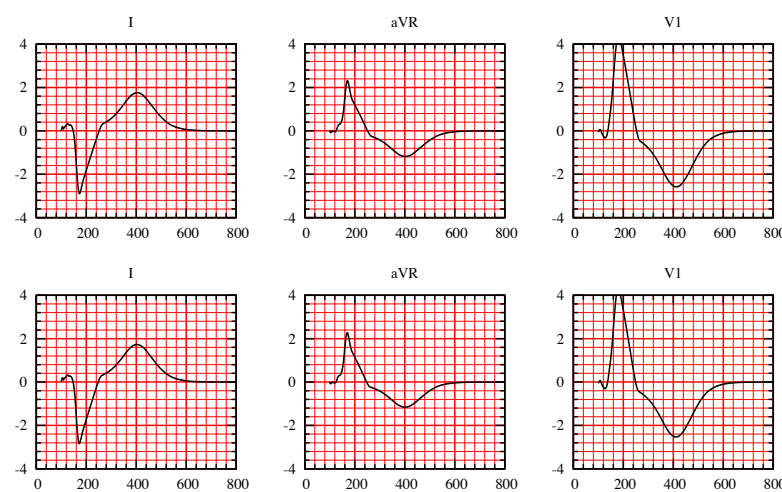

avr

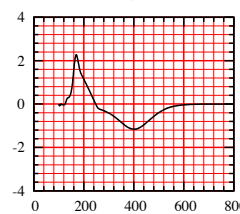

v1

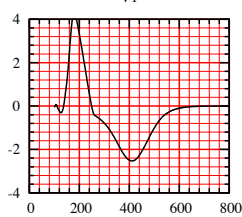

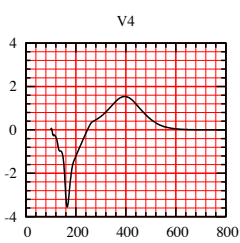

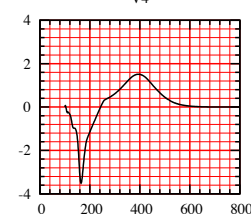

Figure 13: Simulated ECG for a RBBB pathology with heart-torso uncoupling: monodomain (top) and bidomain (bottom) models.

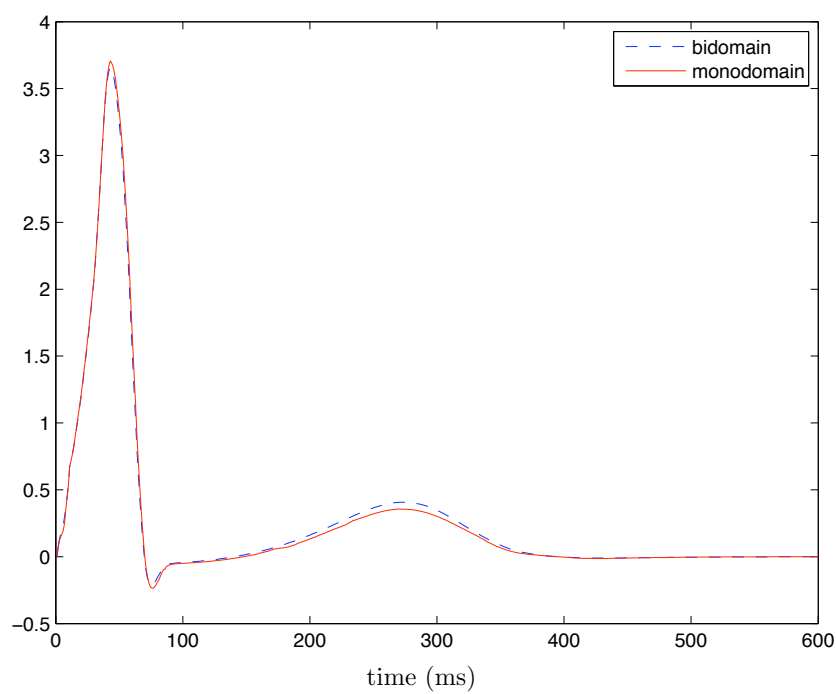

Figure 14: First ECG lead: bidomain and monodomain models with heart-torso uncoupling

\subsection{Isotropy}

The impact of the conductivity anisotropy on the ECG signals is now investigated. To this aim, the numerical simulations of section 4.1 are reconsidered with isotropic conductivities, by setting

$$
\boldsymbol{\sigma}_{\mathrm{e}}^{\mathrm{t}}=\boldsymbol{\sigma}_{\mathrm{e}}^{\mathrm{l}}=3.0 \times 10^{-3} \mathrm{~S} \mathrm{~cm}^{-1}, \quad \boldsymbol{\sigma}_{\mathrm{i}}^{\mathrm{t}}=\boldsymbol{\sigma}_{\mathrm{i}}^{\mathrm{l}}=3.0 \times 10^{-3} \mathrm{~S} \mathrm{~cm}^{-1} .
$$

Figure 15 (top) shows the corresponding ECG signals. The QRS and T waves have the same polarity than in the anisotropic case, Figure 15 (bottom). However, we can clearly observe that the QRS-complex has a smaller duration and that the S-wave amplitude, in leads I and V4, is larger. The impact of anisotropy 

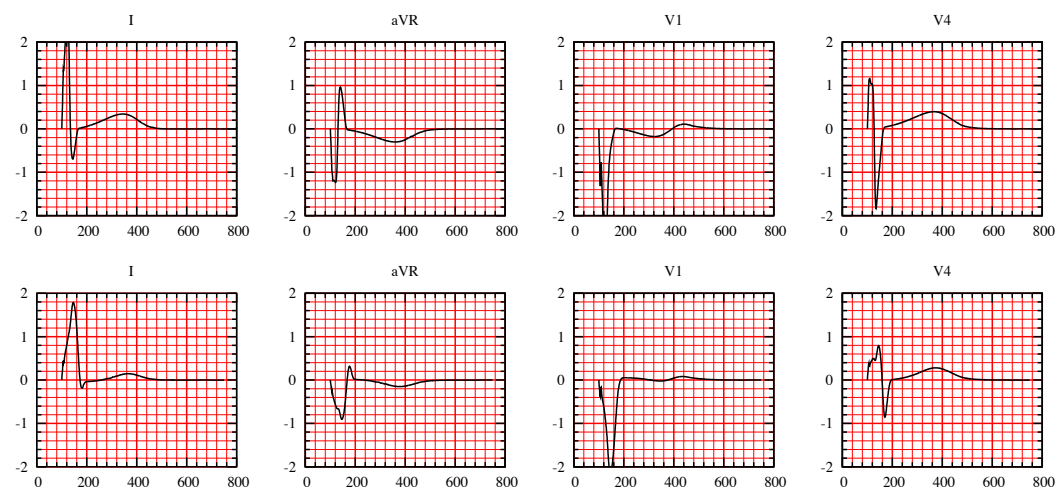

aVR

$\mathrm{v} 1$

$\mathrm{v} 4$
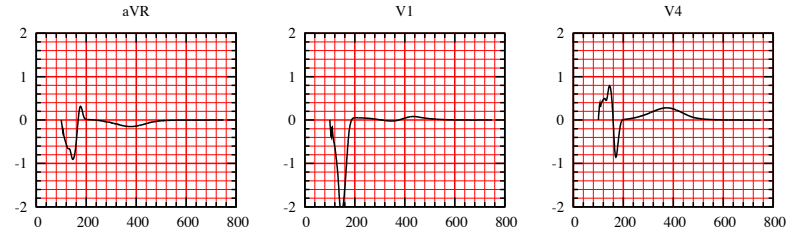

Figure 15: ECG signals: isotropic conductivities (top), anisotropic conductivities (bottom).

is much more striking when dealing with pathological activations. In Figure 16 for instance, the simulated ECG signals for a RBBB pathology have been reported with anisotropic and isotropic conductivities. Notice that the electrical signal is significantly distorted. In particular, the amplitude of the QRS complex is larger in the isotropic case (this observation also holds in the healthy case).

These numerical simulations show that anisotropy has a major impact on the accuracy of ECG signals. Meaningful ECG simulations have therefore to incorporate this modeling feature (see also [11]).

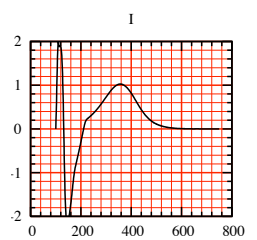

I

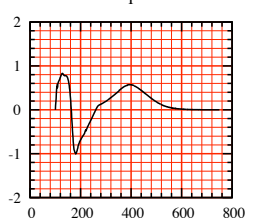

aVR

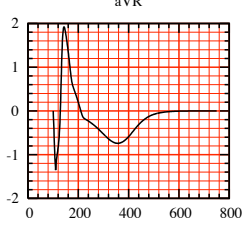

aVR

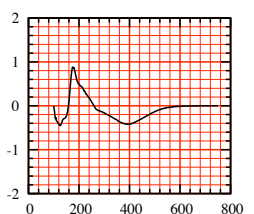

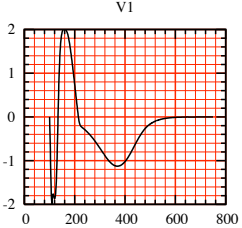

v1

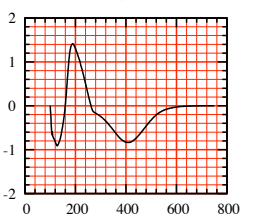

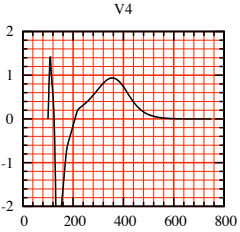

$\mathrm{V} 4$

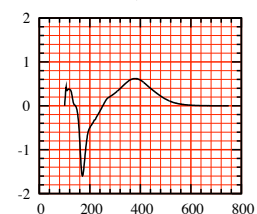

Figure 16: Isotropic (top) and anisotropic (bottom) conductivities in a pathological case (RBBB).

\subsection{Cell homogeneity}

As mentioned in subsection 4.1.5 an heterogeneous coefficient $\tau_{\text {close }}$ has been considered in RS to incorporate an APD gradient across the left ventricle transmural direction. In this paragraph, the myocardium is assumed to have homo- 
geneous cells. The ECG signals corresponding to a constant APD in the whole heart, obtained with $\tau_{\text {close }}=140 \mathrm{~ms}$, are reported in Figure 17 .
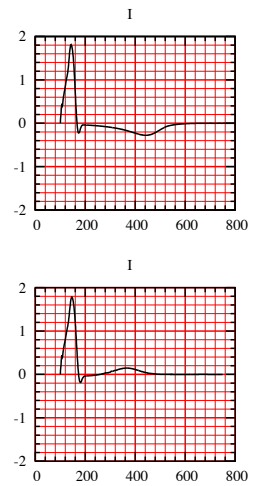

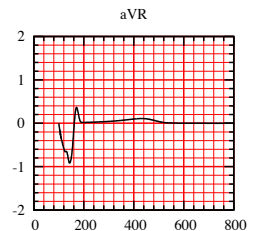

$\mathrm{aVR}$

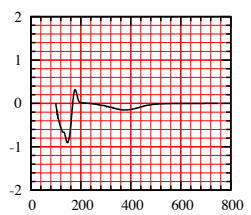

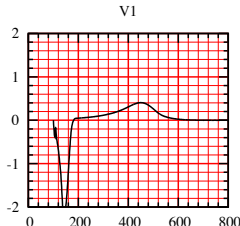

$\mathrm{V} 1$

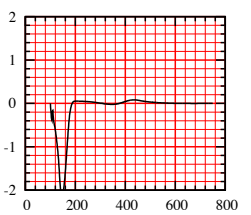

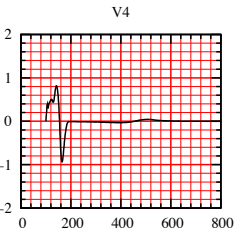

$\mathrm{v} 4$

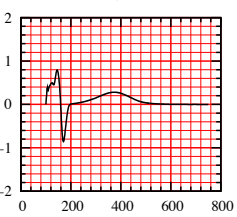

Figure 17: ECG signals: homogeneous action potential duration (top), heterogeneous action potential duration (bottom).

Note that now, in the bipolar lead (I), the T-wave has an opposite polarity with respect to the $\mathbf{R S}$ and to what is usually observed in normal ECGs. Indeed, without transmural APD heterogeneity, the repolarization and the depolarization waves travel in the same direction, which leads to the discordant polarity, between the QRS and the T waves, observed in lead I. On the contrary, the unipolar leads (aVR, V1 and V4) present a similar polarity, irrespectively of the ADP heterogeneity (see also [11]).

As a result, as also noticed in [41, 40, 30, 6], transmural APD heterogeneity is a major ingredient in the simulation of a complete 12-lead ECG with physiological T-wave polarities.

\subsection{Capacitive and resistive effect of the pericardium}

The coupling conditions (2.10) are formally obtained in 31 using an homogenization procedure. In that reference, a perfect electrical coupling is assumed between the heart and the surrounding tissues.

It might be interesting to consider more general coupling conditions. For instance, by assuming that the pericardium (the double-walled sac containing the heart) might induce a resistor-capacitor effect. This can be a way to model pathological conditions - e.g. pericarditis, when the pericardium becomes inflamed - or to take into account the fact that, even in a healthy situation, the heart-torso coupling can be more complex. Thus, we propose to generalize (2.10), by introducing the following resistor-capacitor (R-C) coupling conditions:

$$
\left\{\begin{array}{rlrl}
R_{\mathrm{p}} \boldsymbol{\sigma}_{\mathrm{T}} \boldsymbol{\nabla} u_{\mathrm{T}} \cdot \boldsymbol{n} & =R_{\mathrm{p}} C_{\mathrm{p}} \frac{\partial\left(u_{\mathrm{e}}-u_{\mathrm{T}}\right)}{\partial t}+\left(u_{\mathrm{e}}-u_{\mathrm{T}}\right), & & \text { on } \Sigma, \\
\boldsymbol{\sigma}_{\mathrm{e}} \boldsymbol{\nabla} u_{\mathrm{e}} \cdot \boldsymbol{n} & =\boldsymbol{\sigma}_{\mathrm{T}} \boldsymbol{\nabla} u_{\mathrm{T}} \cdot \boldsymbol{n}, & \text { on } \Sigma,
\end{array}\right.
$$

where $C_{\mathrm{p}}$ and $R_{\mathrm{p}}$ stand for the capacitance and resistance of the pericardium, respectively. Note that, the classical relations 2.10 can be recovered from (5.37) by setting $R_{\mathrm{p}}=0$. To the best of our knowledge, the resistor-capacitor 


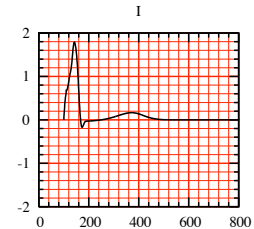

II

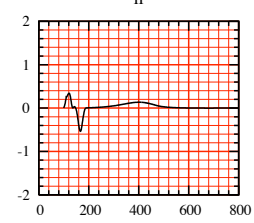

III

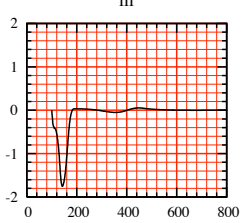

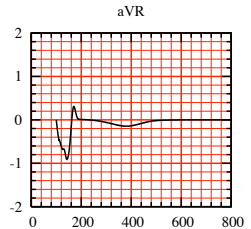

aVL

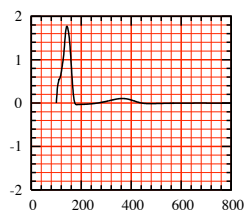

aVF

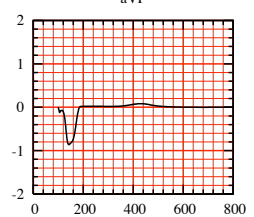

V1

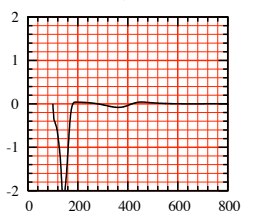

$\mathrm{v} 2$

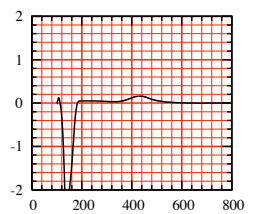

v3

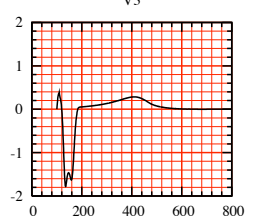

V4

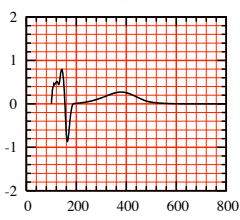

v5

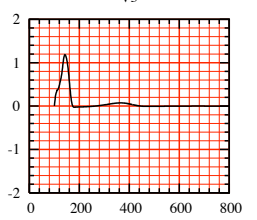

v6

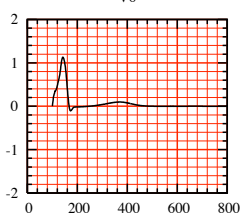

Figure 18: Simulated 12-lead ECG signals: R-C heart-torso coupling conditions with $R_{\mathrm{p}}=10^{2} \Omega \mathrm{cm}^{2}, C_{\mathrm{p}}=0 \mathrm{mF} \mathrm{cm}$.

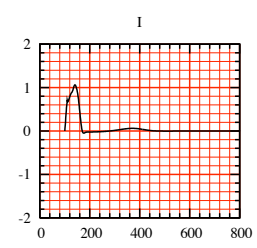

II

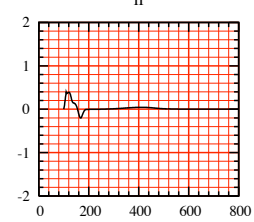

III

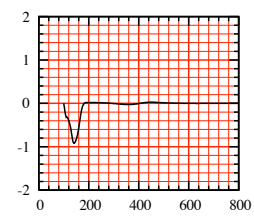

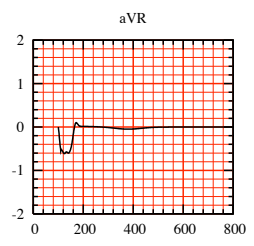

aVL

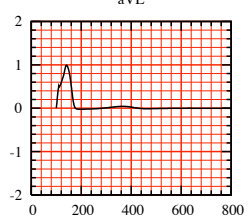

$\mathrm{aVF}$

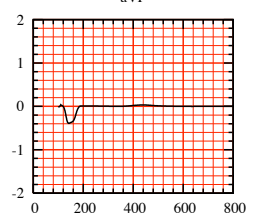

v1

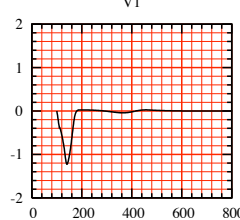

$\mathrm{v} 2$

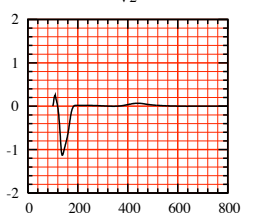

v3

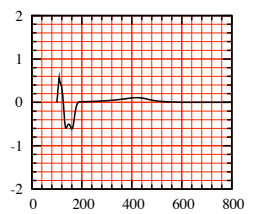

$\mathrm{v} 4$
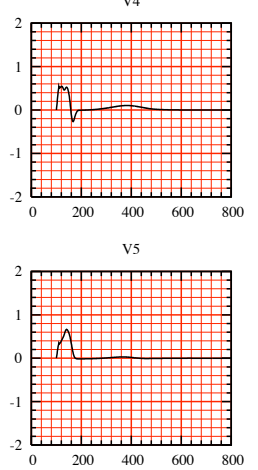

v6

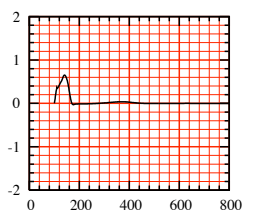

Figure 19: Simulated 12-lead ECG signals: R-C heart-torso coupling conditions with $R_{\mathrm{p}}=10^{4} \Omega \mathrm{cm}^{2}, C_{\mathrm{p}}=0 \mathrm{mF} \mathrm{cm} \mathrm{cm}^{-2}$.

behavior (5.37) of the pericardium is not documented in the literature, so we propose to study its effect on ECGs through numerical simulations.

Numerical tests showed that for $R_{\mathrm{p}}$ small $\left(R_{\mathrm{p}}<10^{3} \Omega \mathrm{cm}^{2}\right.$ approximatively) or $C_{\mathrm{p}}$ large $\left(C_{\mathrm{p}}>1 \mathrm{mF} \mathrm{cm} \mathrm{cm}^{-2}\right.$ approximatively) the simulated ECG is very close 


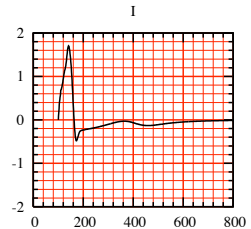

II

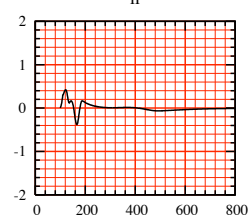

III

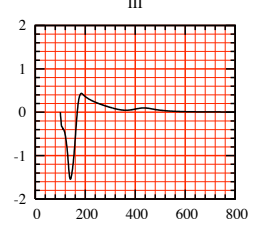

aVR

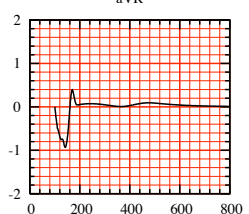

aVL

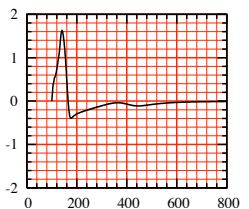

avF

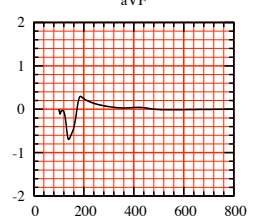

$\mathrm{V} 1$

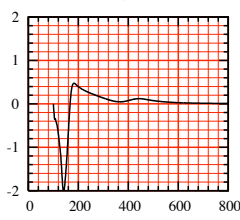

$\mathrm{v} 2$

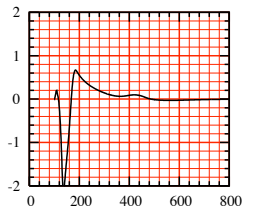

v3

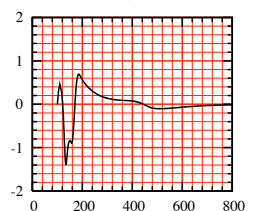

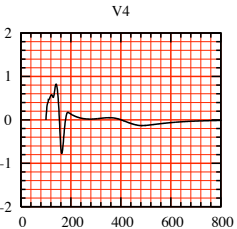

v5

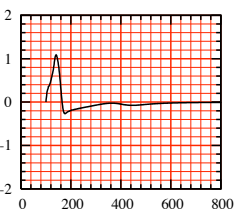

v6

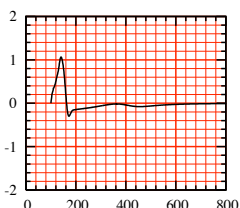

Figure 20: Simulated 12-lead ECG signals: R-C heart-torso coupling conditions with $R_{\mathrm{p}}=10^{20} \Omega \mathrm{cm}^{2}, C_{\mathrm{p}}=10^{-2} \mathrm{mF} \mathrm{cm}^{-2}$

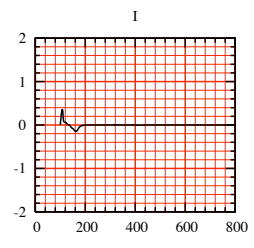

II

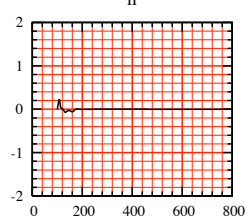

III

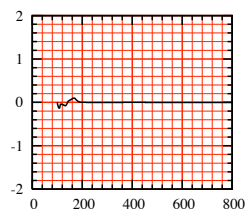

aVR

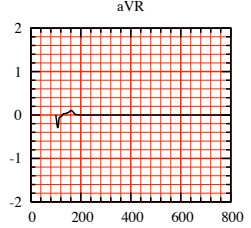

aVL

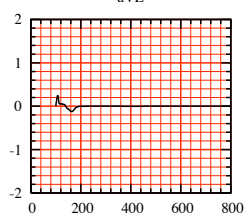

avF

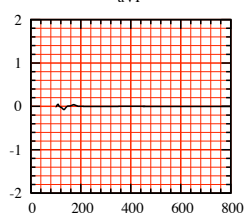

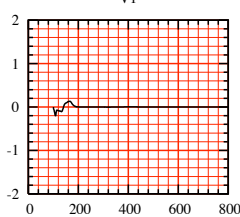

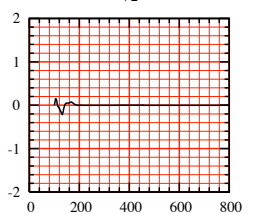

v3

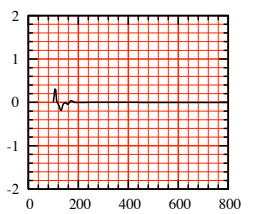

$\mathrm{V} 4$

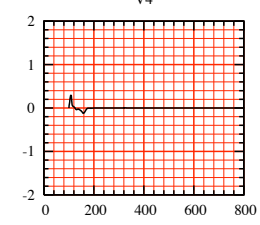

v5

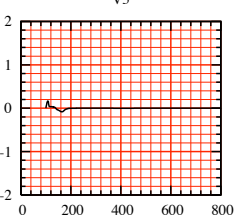

v6

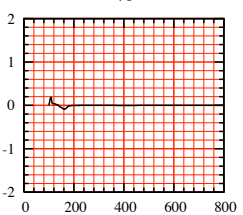

Figure 21: Simulated 12-lead ECG signals: R-C heart-torso coupling conditions with $R_{\mathrm{p}}=10^{20} \Omega \mathrm{cm}^{2}, C_{\mathrm{p}}=10^{-4} \mathrm{mF} \mathrm{cm}{ }^{-2}$.

to the RS. Figure 18, for instance, presents the ECG signals obtained with $R_{\mathrm{p}}=10^{2} \Omega \mathrm{cm}^{2}$ and $C_{\mathrm{p}}=0 \mathrm{mF} \mathrm{\textrm {cm } ^ { 2 }}$.

In order to illustrate the resistor effect, we have reported in Figure 19 the ECG obtained with $C_{\mathrm{p}}=0 \mathrm{mF} \mathrm{cm} \mathrm{cm}^{-2}$ and $R_{\mathrm{p}}=10^{4} \Omega . \mathrm{cm}^{2}$. We clearly observe 
that the amplitude of the signals is smaller than in the RS. More generally, this amplitude decreases when $R_{\mathrm{p}}$ increases, as expected.

We now focus on the capacitor effect by taking $R_{\mathrm{p}}$ very large. Figure 20 presents the ECG signals obtained with $R_{\mathrm{p}}=10^{20} \Omega \mathrm{cm}^{2}$ and $C_{\mathrm{p}}=10^{-2} \mathrm{mF} \mathrm{cm}^{-2}$. We observe that the capacitive term induces a relaxation effect and distorts the signal. In particular, the T-wave is inverted in all the ECG leads and the S-wave duration is larger than for the RS. At last, Figure 21] shows that for very small values of $C_{\mathrm{p}}$ the amplitude of the ECG is also very small. This can be formally explained by the fact that, in this case, condition $(5.37)_{1}$ approximately becomes $\boldsymbol{\sigma}_{\mathrm{T}} \boldsymbol{\nabla} u_{\mathrm{T}} \cdot \boldsymbol{n}=0$ on $\Sigma$ : no heart information is transferred to the torso, leading to very low ECG signals.

\section{Numerical investigations with weak heart-torso coupling}

In this section, we investigate the ECG sensitivity to the time and space discretizations and to the heart and torso model parameters. To carry out these studies at a reasonable computational cost, we consider the heart-torso uncoupling. Although we have noticed (in section 5.1) that uncoupling may affect the ECG accuracy in some cases, we can expect that the conclusions of the sensitivity analysis remain still valid under this simplification.

\subsection{Time and space convergence}

In this section, we are not interested in the convergence of the whole solution of the $\mathbf{R M}$ with respect to the space and time discretization parameters, but rather in the convergence of the ECG which is here considered as the quantity of interest.

\subsubsection{Time convergence}

In Figure 22, we present the first ECG lead (lead I) obtained for three different time-step sizes $\delta t=0.25,0.5$ and $2 \mathrm{~ms}$. The $l^{2}$-norm of the relative difference with the result obtained with $\delta t=0.25 \mathrm{~ms}$ is $10 \%$ when $\delta t=2 \mathrm{~ms}$ and $2.0 \%$ when $\delta t=0.5 \mathrm{~ms}$.

\subsubsection{Space convergence}

Three different levels of refinements are considered for the heart and the torso meshes, as shown in Table 5. The finite element meshes used in the RS are the R2. In Figure 23, we report the first lead of the ECGs obtained for these simulations.

Although the whole solution might not be fully converged within the heart, we can observe that the quantity of interest - namely the ECG - is almost unaffected by the last refinement. Therefore, in a goal-oriented refinement framework, the solution may indeed be considered as converged. 


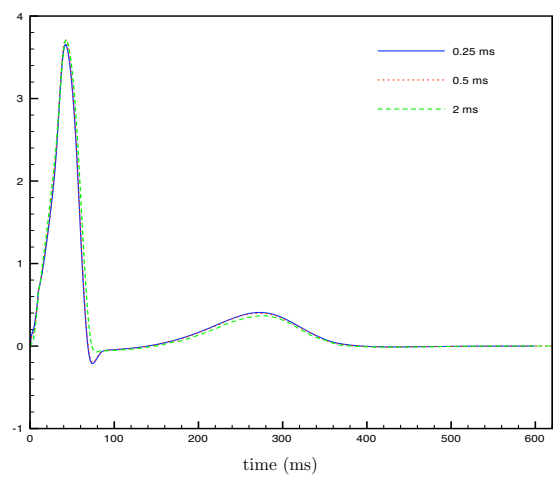

Figure 22: Comparison of three simulations of ECG (lead I) with three different time steps: $2,0.5$ and $0.25 \mathrm{~ms}$

\begin{tabular}{|c|c|c|c|}
\hline Meshes & Heart nodes & Torso nodes & Total number of tetrahedra \\
\hline R1 & 13000 & 56000 & 370000 \\
\hline R2 & 80000 & 120000 & 1080000 \\
\hline R3 & 236000 & 232000 & 2524000 \\
\hline
\end{tabular}

Table 5: There different levels of refinement for the computational heart and torso meshes (rounded off values).

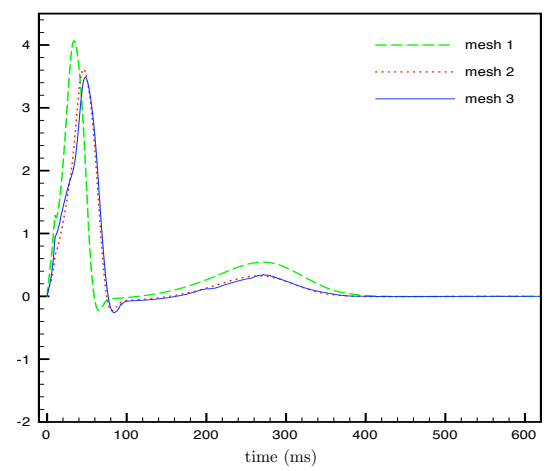

Figure 23: Comparison of three simulations of ECG (lead I), using three different levels of mesh refinement (see Table 5).

\subsection{Sensitivity to model parameters}

In this section, we study the sensitivity of ECG to some model parameters. This is fundamental step prior to addressing its estimation (see e.g. [5]) using data assimilation techniques.

Suppose that $\alpha_{1}, \alpha_{2}, \ldots, \alpha_{\mathrm{p}}$ are parameters the ECG depends upon, i.e.

$$
E C G=E C G\left(\alpha_{1}, \alpha_{2}, \ldots, \alpha_{\mathrm{p}}\right)
$$


The ECG sensitivity to parameter $\alpha_{i}$ can then be approximated as

$\partial_{\alpha_{i}} E C G\left(\alpha_{1}, \alpha_{2}, \ldots, \alpha_{\mathrm{p}}\right) \approx \frac{E C G\left(\alpha_{1}, \alpha_{2}, \ldots,(1+\epsilon) \alpha_{i}, \ldots, \alpha_{\mathrm{p}}\right)-E C G\left(\alpha_{1}, \alpha_{2}, \ldots, \alpha_{\mathrm{p}}\right)}{\epsilon \alpha_{i}}$,

where $\epsilon$ is a small parameter, in our case $10^{-6} \leq \epsilon \leq 10^{-4}$ gives a good approximation. Instead of $\partial_{\alpha_{i}} E C G\left(\alpha_{1}, \alpha_{2}, \ldots, \alpha_{\mathrm{p}}\right)$ we consider the normalized value $\alpha_{i} \partial_{\alpha_{i}} E C G\left(\alpha_{1}, \alpha_{2}, \ldots, \alpha_{\mathrm{p}}\right)$, which allows to compare the sensitivity irrespectively of the parameter scales. In the next paragraphs, we provide time evolution of this scaled derivative, evaluated around the parameters used in the RS. Once more, for the sake of conciseness, we focus on the first ECG lead.

\subsubsection{Ionic model parameters}

In this paragraph, we investigate the sensitivity of the ECG to the MitchellSchaeffer parameters. In Figure 24, we have reported the normalized derivatives with respect to $\tau_{\text {in }}, \tau_{\text {out }}, \tau_{\text {open }}$ or $\tau_{\text {close }}$. The high ECG sensitivity to $\tau_{\text {in }}$ is clearly visible, particularly during the QRS-complex. The sensitivity to $\tau_{\text {out }}$ is moderate both during the depolarization and depolarization phases. As expected, the sensitivity to $\tau_{\text {close }}$ is only relevant during repolarization. Interestingly, the sensitivity to $\tau_{\text {open }}$ is relatively small. Therefore, this parameter may be removed (i.e. keep fixed) within an inverse estimation procedure.

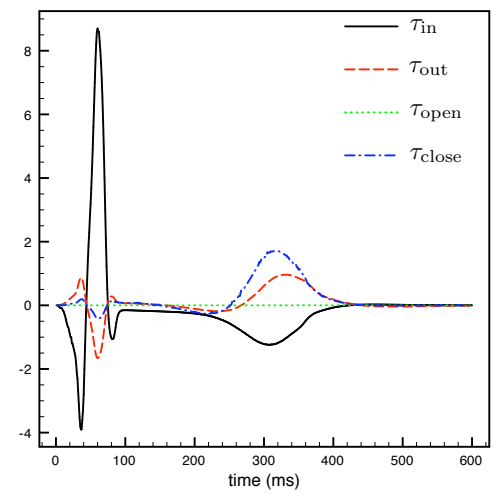

Figure 24: Normalized ECG sensitivity to $\tau_{\text {in }}, \tau_{\text {out }}, \tau_{\text {open }}$ and $\tau_{\text {close }}$.

\subsubsection{Bidomain model parameters}

We first focus on the ECG sensitivity to the local myocardium conductivities: $\sigma_{\mathrm{e}}^{\mathrm{t}}, \sigma_{\mathrm{e}}^{\mathrm{l}}, \sigma_{\mathrm{i}}^{\mathrm{t}}$ and $\sigma_{\mathrm{i}}^{\mathrm{l}}$. The corresponding normalized derivatives are given in Figure 25. During depolarization (QRS-complex), the ECG is mainly sensitive to transverse conductivity $\left(\sigma_{\mathrm{e}}^{\mathrm{t}}, \sigma_{\mathrm{i}}^{\mathrm{t}}\right)$. This can be due to the dominating transmural propagation of the depolarization wave in the left ventricle (see Figure 4 (left)). During repolarization (T-wave), on the contrary, the ECG shows approximately the same sensitivity to all the local conductivities.

We now pursue our sensitivity analysis, by considering the parameters $A_{\mathrm{m}}$ and $C_{\mathrm{m}}$. The corresponding normalized derivatives are given in Figure 26. We 


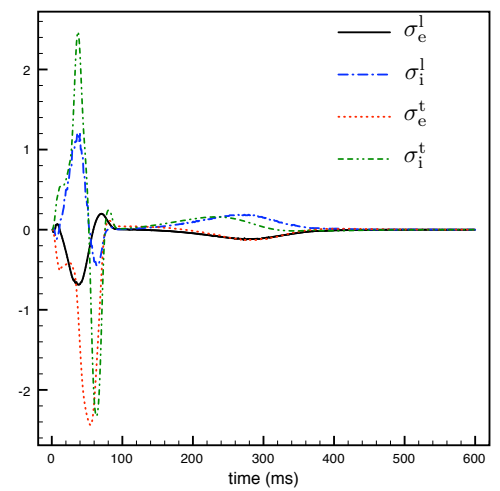

Figure 25: Normalized ECG sensitivity to the local myocardium conductivities: $\sigma_{\mathrm{e}}^{\mathrm{t}}, \sigma_{\mathrm{e}}^{\mathrm{l}}, \sigma_{\mathrm{i}}^{\mathrm{t}}$ and $\sigma_{\mathrm{i}}^{\mathrm{l}}$.

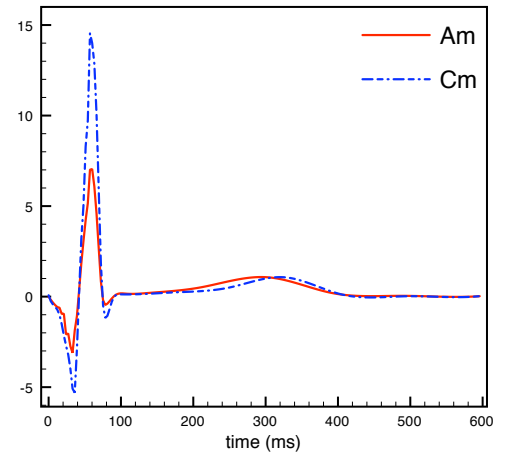

Figure 26: Normalized ECG sensitivity to $A_{\mathrm{m}}$ and $C_{\mathrm{m}}$.

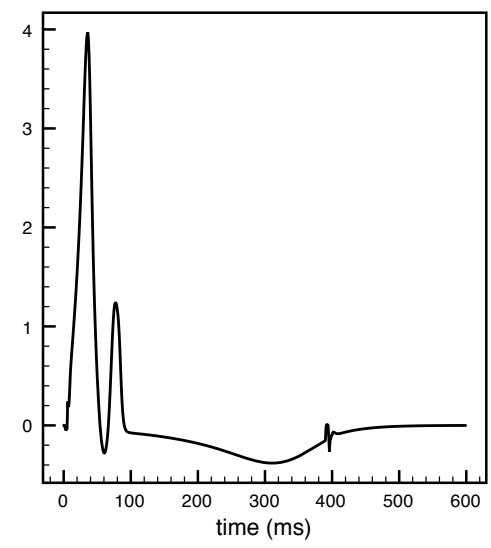

Figure 27: Normalized ECG sensitivity to the activation angular velocity.

observe a strong sensitivity to both parameters during depolarization. Whereas, during the repolarization phase, the sensitivity is reduced. 
At last, we investigate the sensitivity of the ECG to the initial activation in the heart (see Appendix A. More precisely, we focus on the sensitivity to the activation angular velocity $\frac{\pi}{2 t_{2}}$. The corresponding normalized derivative is reported Figure 27. As expected, the ECG is strongly sensitive to this parameter, particularly during the depolarization phase.

\subsubsection{Torso parameters}

We finally consider the sensitivity of the ECG to the torso conductivities $\sigma_{\mathrm{T}}^{\mathrm{l}}, \sigma_{\mathrm{T}}^{\mathrm{b}}$ and $\sigma_{\mathrm{T}}^{\mathrm{t}}$. Note that, in a heart-torso uncoupling framework, the corresponding three normalized derivatives are linked by a linear relation. Indeed, from (2.11) and (5.20), we have that, for all $\lambda \in \mathbb{R}, u_{\mathrm{T}}$ solves

$$
\left\{\begin{array}{rll}
\operatorname{div}\left(\lambda \boldsymbol{\sigma}_{\mathrm{T}} \boldsymbol{\nabla} u_{\mathrm{T}}\right)=0, & \text { in } & \Omega_{\mathrm{T}}, \\
u_{\mathrm{T}}=u_{\mathrm{e}}, & \text { on } & \Sigma, \\
\lambda \boldsymbol{\sigma}_{\mathrm{T}} \boldsymbol{\nabla} u_{\mathrm{T}} \cdot \boldsymbol{n}_{\mathrm{T}}=0, & \text { on } & \Gamma_{\mathrm{ext}} .
\end{array}\right.
$$

In other words,

$$
u_{\mathrm{T}}\left(\lambda \sigma_{\mathrm{T}}^{\mathrm{l}}, \lambda \sigma_{\mathrm{T}}^{\mathrm{b}}, \lambda \sigma_{\mathrm{T}}^{\mathrm{t}}\right)=u_{\mathrm{T}}\left(\sigma_{\mathrm{T}}^{\mathrm{l}}, \sigma_{\mathrm{T}}^{\mathrm{b}}, \sigma_{\mathrm{T}}^{\mathrm{t}}\right) .
$$

Differentiating this relation with respect to $\lambda$ (and evaluating the resulting expression at $\lambda=1$ ) yields

$$
\sigma_{\mathrm{T}}^{\mathrm{l}} \partial_{\sigma_{\mathrm{T}}^{\mathrm{l}}} u_{\mathrm{T}}+\sigma_{\mathrm{T}}^{\mathrm{b}} \partial_{\sigma_{\mathrm{T}}^{\mathrm{b}}} u_{\mathrm{T}}+\sigma_{\mathrm{T}}^{\mathrm{t}} \partial_{\sigma_{\mathrm{T}}^{\mathrm{t}}} u_{\mathrm{T}}=0 .
$$

Thus, from (4.17), we obtain a similar relation for the normalized ECG derivatives:

$$
\sigma_{\mathrm{T}}^{\mathrm{l}} \partial_{\sigma_{\mathrm{T}}^{1}} E C G+\sigma_{\mathrm{T}}^{\mathrm{b}} \partial_{\sigma_{\mathrm{T}}^{\mathrm{b}}} E C G+\sigma_{\mathrm{T}}^{\mathrm{t}} \partial_{\sigma_{\mathrm{T}}^{\mathrm{t}}} E C G=0 .
$$

Figure 28 presents the normalized derivatives of the ECG with respect to the tissue, lung and bone conductivities. This figure clearly shows that the ECG

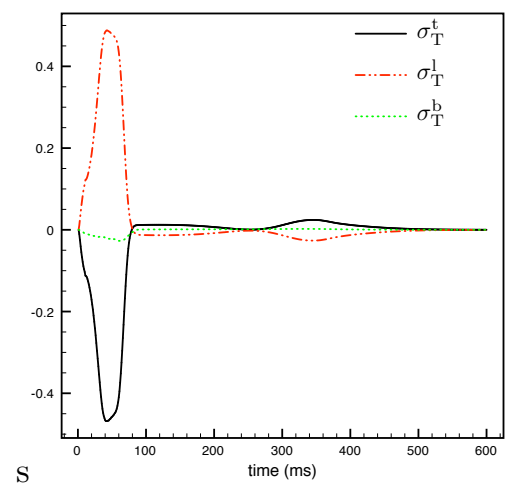

Figure 28: Normalized ECG sensitivity to $\sigma_{\mathrm{T}}^{\mathrm{l}}, \sigma_{\mathrm{T}}^{\mathrm{b}}$ and $\sigma_{\mathrm{T}}^{\mathrm{t}}$.

sensitivity to the bone parameter $\sigma_{\mathrm{T}}^{\mathrm{b}}$ is negligible compared to its sensitivity to the tissue and lung parameters. Thus, if we have in mind to limit the number of parameters to be estimated, $\sigma_{\mathrm{T}}^{\mathrm{b}}$ can safely be fixed to the value used in the RS. 


\section{Conclusion}

A fully PDE/ODE based mathematical model for the numerical simulation of ECGs has been described. The electrical activity of the heart is based on the coupling of the bidomain equations with the Mitchell-Schaeffer phenomenological ionic model, including anisotropic conductivities and transmural APD heterogeneity. This system of equations has been coupled to a generalized Laplace equation in the torso, with inhomogeneous conductivity (bone, lungs and remaining tissue). A detailed description of the different algorithms used for the numerical solution of the resulting ECG model has been also provided.

Our approach has several limitations: we did not consider the atria, which prevents us from computing the $\mathrm{P}$ wave of the ECG; the cell model being phenomenological, it cannot handle complex ionic interactions; the effect of the blood flow on the ECG was neglected; the geometry of the ventricles were simplified.

Despite the above mentioned limitations, we were able to compute a satisfactory healthy 12-lead ECG, with a limited number a parameters. To the best of our knowledge, this constitutes a breakthrough in the modeling of ECGs with partial differential equations. Moreover, for a pathological situation corresponding to a bundle branch block, our simulations have provided an ECG which satisfies the typical criteria used by medical doctors to detect this pathology. This shows, in particular, that our numerical model have some predictive features.

In a second part, we have studied the impact of some modeling assumptions on the ECGs. The main conclusions of this investigation are the following:

1. As far as the general shape of the ECGs is concerned, heart-torso uncoupling can be considered. The level of accuracy obtained with uncoupling is probably sufficient in several applications, which may explain why this simplification is so widespread in the literature. Nevertheless, our numerical results have clearly pointed out that the amplitudes of the ECG signals obtained via uncoupling and full coupling can significantly differ. We therefore recommend to carefully check in each specific situations whether the uncoupling approximation is acceptable or not.

2. In agreement with other studies, we noticed that cell heterogeneity and fiber anisotropy have an important impact on the ECG and, therefore, cannot be neglected.

3. The bidomain equations can apparently be safely replaced by the monodomain equations without significantly affecting the ECG. Nevertheless, even with this simplification, we point out that the transmembrane potential $V_{\mathrm{m}}$ and the extracellular potential $u_{\mathrm{e}}$ still have to be solved simultaneously when the heart and the torso are fully coupled. To be really attractive, the monodomain simplification has therefore to come with the uncoupling approximation, which can affect the ECG, as mentioned above.

4. We have proposed a new heart-torso coupling condition which takes into account possible capacitive and resistive effects of the pericardium. We did not find in the literature any evidence of these effects and our results show that it does not seem necessary to include them in order to get realistic 
healthy ECGs. Nevertheless, these coupling conditions might be relevant in some pathologies affecting the pericardial sac and the simulations we provided to illustrate these effects might be useful for future works.

5. At last, a sensitivity analysis has shown that the most critical parameters of the bidomain model are $C_{\mathrm{m}}, A_{\mathrm{m}}$, the angular velocity of the activation wave and the transverse conductivities $\sigma_{\mathrm{i}}^{\mathrm{t}}$ and $\sigma_{\mathrm{e}}^{\mathrm{t}}$. As regards the ECG sensitivity to the ionic model parameters, we have noticed a extreme sensitivity of the QRS-complex to the parameter $\tau_{\text {in }}$ and a high sensitivity of the T-wave to the parameter $\tau_{\text {close }}$. Moreover, we have also observed that the ECG sensitivity to the torso conductivity parameters is less significant than to the heart model parameters.

To conclude, our main concern during this study was to build a model rich enough to provide realistic ECGs and simple enough to be easily parametrized. In spite of its shortcomings, the proposed approach essentially fulfills these requirements and is therefore a good candidate to address inverse problems. This will be investigated in future works.

\section{A External stimulus}

In order to initiate the spread of excitation within the myocardium, we apply a given volume current density to a thin subendocardial layer of the ventricles during a small period of time $t_{\text {act }}$. In the left ventricle, this thin layer $(1.6 \mathrm{~mm})$

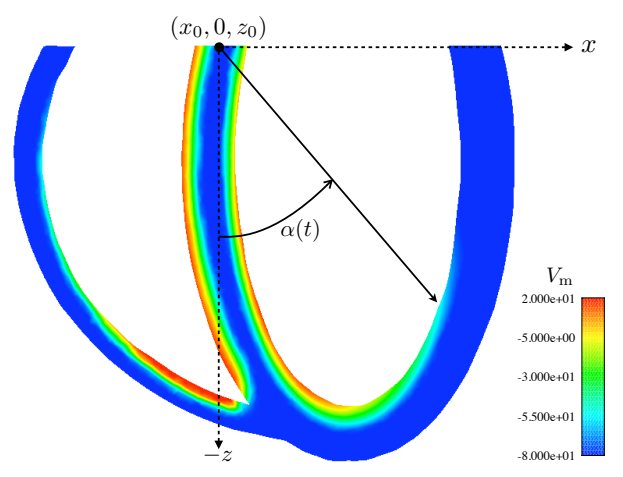

Figure 29: Geometrical description of the external stimulus (plane cut $y=0$ ).

of external activation is given by

$$
S \stackrel{\text { def }}{=}\left\{(x, y, z) \in \Omega_{\mathrm{H}} / c_{1} \leq a x^{2}+b y^{2}+c z^{2} \leq c_{2}\right\},
$$

where $a, b, c, c_{1}$ and $c_{2}$ are given constants, with $c_{1}<c_{2}$, see Figure 29 . The source current $I_{\text {app }}$, involved in (2.7), is then parametrized as follows:

$$
I_{\text {app }}(x, y, z, t)=I_{0}(x, y, z) \chi_{S}(x, y, z) \chi_{\left[0, t_{\text {act }}\right]}(t) \psi(x, z, t),
$$

where

$$
I_{0}(x, y, z) \stackrel{\text { def }}{=} i_{\text {app }}\left[\frac{c_{2}}{c_{2}-c_{1}}-\frac{1}{c_{2}-c_{1}}\left(a x^{2}+b y^{2}+c z^{2}\right)\right],
$$


with $i_{\text {app }}$ the amplitude of the external applied stimulus,

$$
\begin{gathered}
\chi_{S}(x, y, z) \stackrel{\text { def }}{=}\left\{\begin{array}{lll}
1 & \text { if } & (x, y, z) \in S, \\
0 & \text { if } & (x, y, z) \notin S,
\end{array}\right. \\
\chi_{\left[0, t_{\mathrm{act}}\right]}(t) \stackrel{\text { def }}{=}\left\{\begin{array}{lll}
1 & \text { if } & t \in\left[0, t_{\mathrm{act}}\right], \\
0 & \text { if } & t \notin\left[0, t_{\mathrm{act}}\right],
\end{array}\right. \\
\psi(x, z, t) \stackrel{\text { def }}{=}\left\{\begin{array}{lll}
1 & \text { if } & \operatorname{atan}\left(\frac{x-x_{0}}{z-z_{0}}\right) \leq \alpha(t), \\
0 & \text { if } & \operatorname{atan}\left(\frac{x-x_{0}}{z-z_{0}}\right)>\alpha(t),
\end{array}\right.
\end{gathered}
$$

the activated angle $\alpha(t) \stackrel{\text { def }}{=} \frac{t \pi}{2 t_{\text {act }}}$ and $t_{\text {act }}=10 \mathrm{~ms}$. The activation current in the right ventricle is built in a similar fashion.

\section{Acknowledgements}

This work was partially supported by INRIA through its large scope initiative CardioSense3D. The authors wish to thank Elsie Phé (INRIA) for her work on the anatomical models and meshes, and Michel Sorine (INRIA) for valuable discussions regarding, in particular, the heart-torso transmission conditions.

\section{References}

[1] B. Aehlert. ECGs Made Easy. Mosby Jems, Elsevier, third edition, 2006.

[2] C. Antzelevitch. Cellular basis for the repolarization waves of the ECG. Ann. N. Y. Acad. Sci., 1080:268-281, 2006.

[3] R.C. Barr, M.3rd Ramsey, and M.S. Spach. Relating epicardial to body surface potential distributions by means of transfer coefficients based on geometry measurements. IEEE Trans. Biomed. Eng., 24(1):1-11, 1977.

[4] G. Beeler and H. Reuter. Reconstruction of the action potential of ventricular myocardial fibres. J. Physiol. (Lond.), 268:177-210, 1977.

[5] M. Boulakia, M. A. Fernández, J. F. Gerbeau, and N. Zemzemi. Direct and inverse problems in electrocardiography. AIP Conference Proceedings, 1048(1):113-117, 2008.

[6] M. Boulakia, M.A. Fernández, J.-F. Gerbeau, and N. Zemzemi. Towards the numerical simulation of electrocardiograms. In F.B. Sachse and G. Seemann, editors, Functional Imaging and Modeling of the Heart, volume 4466 of Lecture Notes in Computer Science, pages 240-249. Springer-Verlag, 2007.

[7] M. Boulakia, M.A. Fernández, J.-F. Gerbeau, and N. Zemzemi. A coupled system of PDEs and ODEs arising in electrocardiograms modelling. Applied Math. Res. Exp., 2008(abn002):28, 2008. 
[8] M. Buist and A. Pullan. Torso coupling techniques for the forward problem of electrocardiography. Ann. Biomed. Eng., 30(10):1299-1312, 2002.

[9] J. Clements, J. Nenonen, P.K.J. Li, and B.M. Horacek. Activation dynamics in anisotropic cardiac tissue via decoupling. Annals of Biomedical Engineering, 32(7):984-990, 2004.

[10] P. Colli Franzone and L.F. Pavarino. A parallel solver for reaction-diffusion systems in computational electrocardiology. Math. Models Methods Appl. Sci., 14(6):883-911, 2004.

[11] P. Colli Franzone, L.F. Pavarino, S. Scacchi, and B. Taccardi. Effects of anisotropy and transmural heterogeneity on the T-wave polarity of simulated electrograms. In N. Ayache, H. Delingette, and M. Sermesant, editors, Functional Imaging and Modeling of the Heart, volume 5528 of Lecture Notes in Computer Science, pages 513-523. Springer-Verlag, 2009.

[12] P. Colli Franzone, L.F. Pavarino, and B. Taccardi. Simulating patterns of excitation, repolarization and action potential duration with cardiac bidomain and monodomain models. Math. Biosci., 197(1):35-66, 2005.

[13] C.E. Conrath and T. Opthof. Ventricular repolarization: an overview of (patho)physiology, sympathetic effects and genetic aspects. Prog. Biophys. Mol. Biol., 92(3):269-307, Nov 2006.

[14] D. di Bernardo and A. Murray. Modelling cardiac repolarisation for the study of the T wave: effect of repolarisation sequence. Chaos, Solitons $\&$ Fractals, 13(8):1743-1748, 2002.

[15] K. Djabella and M. Sorine. Differential model of the excitation-contraction coupling in a cardiac cell for multicycle simulations. In EMBEC'05, volume 11, pages 4185-4190, Prague, 2005.

[16] G. Ebrard, M.A. Fernández, J.-F. Gerbeau, F. Rossi, and N. Zemzemi. From intracardiac electrograms to electrocardiograms. models and metamodels. In N. Ayache, H. Delingette, and M. Sermesant, editors, Functional Imaging and Modeling of the Heart, volume 5528 of Lecture Notes in Computer Science, pages 524-533. Springer-Verlag, 2009.

[17] M. Ethier and Y. Bourgault. Semi-Implicit Time-Discretization Schemes for the Bidomain Model. SIAM Journal on Numerical Analysis, 46:2443, 2008.

[18] F. Fenton and A. Karma. Vortex dynamics in three-dimensional continuous myocardium with fiber rotation: Filament instability and fibrillation. Chaos, 8(1):20-47, 1998.

[19] R. Fitzhugh. Impulses and physiological states in theoretical models of nerve membrane. Biophys. J., 1:445-465, 1961.

[20] M.R. Franz, K. Bargheer, W. Rafflenbeul, A. Haverich, and P.R. Lichtlen. Monophasic action potential mapping in human subjects with normal electrocardiograms: direct evidence for the genesis of the T wave. Circulation, 75(2):379-386, 1987. 
[21] P. Frey. Yams: A fully automatic adaptive isotropic surface remeshing procedure. Technical report 0252, Inria, Rocquencourt, France, Nov. 2001.

[22] P.L. George, F. Hecht, and E. Saltel. Fully automatic mesh generator for 3d domains of any shape. Impact of Comp. in Sci. ans Eng.,, 2:187-218, 1990.

[23] L. Gerardo-Giorda, L. Mirabella, F. Nobile, M. Perego, and A. Veneziani. A model-based block-triangular preconditioner for the bidomain system in electrocardiology. J. Comp. Phys., 228(10):3625-3639, 2009.

[24] A.L. Goldberger. Clinical Electrocardiography: A Simplified Approach. Mosby-Elsevier, 7th edition, 2006.

[25] L.S. Green, B. Taccardi, P.R. Ershler, and R.L. Lux. Epicardial potential mapping. effects of conducting media on isopotential and isochrone distributions. Circulation, 84(6):2513-2521, 1991.

[26] R.M. Gulrajani. Models of the electrical activity of the heart and computer simulation of the electrocardiogram. Crit. Rev. Biomed. Eng., 16(1):1-6, 1988.

[27] T. Higuchi and Y. Nakaya. T wave polarity related to the repolarization process of epicardial and endocardial ventricular surfaces. American Heart Journal, 108(2):290-295, 1984.

[28] G. Huiskamp. Simulation of depolarization in a membrane-equations-based model of the anisotropic ventricle. EEE Trans. Biomed. Eng., 5045(7):847$855,1998$.

[29] B. Irons and R.C. Tuck. A version of the aitken accelerator for computer implementation. Int. J. Num. Meth. Engng., 1:275-277, 1969.

[30] D.U.J Keller, G. Seemann, D.L. Weiss, D. Farina, J. Zehelein, and O. Dössel. Computer based modeling of the congenital long-qt 2 syndrome in the visible man torso: From genes to ECG. In Proceedings of the 29th Annual International Conference of the IEEE EMBS, pages 1410-1413, 2007.

[31] W. Krassowska and J.C. Neu. Effective boundary conditions for syncitial tissues. IEEE Trans. Biomed. Eng., 41(2):143-150, 1994.

[32] G. T. Lines, M. L. Buist, P. Grottum, A. J. Pullan, J. Sundnes, and A. Tveito. Mathematical models and numerical methods for the forward problem in cardiac electrophysiology. Comput. Visual. Sci., 5(4):215-239, 2003.

[33] C. Luo and Y. Rudy. A dynamic model of the cardiac ventricular action potential. i. simulations of ionic currents and concentration changes. Circ. Res., 74(6):1071-1096, 1994.

[34] C.H. Luo and Y. Rudy. A model of the ventricular cardiac action potential. depolarisation, repolarisation, and their interaction. Circ. Res, 68(6):1501$1526,1991$. 
[35] J. Malmivuo and R. Plonsey. Bioelectromagnetism. Principles and applications of bioelectric and biomagnetic fields. Oxford University Press, New York, 1995.

[36] C.C. Mitchell and D.G. Schaeffer. A two-current model for the dynamics of cardiac membrane. Bulletin Math. Bio., 65:767-793, 2003.

[37] J.C. Neu and W. Krassowska. Homogenization of syncytial tissues. Crit. Rev. Biomed. Eng., 21(2):137-199, 1993.

[38] D. Noble, A. Varghese, P. Kohl, and P. Noble. Improved guinea-pig ventricular cell model incorporating a diadic space, ikr and iks, and lengthand tension-dependent processes. Can. J. Cardiol., 14(1):123-134, 1998.

[39] M. Pennacchio, G. Savaré, and P. Colli Franzone. Multiscale modeling for the bioelectric activity of the heart. SIAM Journal on Mathematical Analysis, 37(4):1333-1370, 2005.

[40] M. Potse, G. Baroudi, P.A. Lanfranchi, and A. Vinet. Generation of the $\mathrm{t}$ wave in the electrocardiogram: lessons to be learned from long-QT syndromes. In Canadian Cardiovascular Congress, 2007.

[41] M. Potse, B. Dubé, and M. Gulrajani. ECG simulations with realistic human membrane, heart, and torso models. In Proceedings of the 25th Annual Intemational Conference of the IEEE EMBS, pages 70-73, 2003.

[42] M. Potse, B. Dubé, J. Richer, A. Vinet, and R. M. Gulrajani. A comparison of monodomain and bidomain reaction-diffusion models for action potential propagation in the human heart. Biomedical Engineering, IEEE Transactions on, 53(12):2425-2435, Dec. 2006.

[43] M. Potse, B. Dubé, and A. Vinet. Cardiac anisotropy in boundaryelement models for the electrocardiogram. Med. Biol. Eng. Comput. DOI:10.1007/s11517-009-0472-x.

[44] A.J. Pullan, M.L. Buist, and L.K. Cheng. Mathematically modelling the electrical activity of the heart: From cell to body surface and back again. World Scientific Publishing Co. Pte. Ltd., Hackensack, NJ, 2005.

[45] A. Quarteroni, R. Sacco, and F. Saleri. Numerical mathematics, volume 37 of Texts in Applied Mathematics. Springer-Verlag, Berlin, second edition, 2007.

[46] A. Quarteroni and A. Valli. Domain decomposition methods for partial differential equations. Numerical Mathematics and Scientific Computation. The Clarendon Press Oxford University Press, New York, 1999. Oxford Science Publications.

[47] F.B. Sachse. Computational Cardiology: Modeling of Anatomy, Electrophysiology, and Mechanics. Springer-Verlag, 2004.

[48] S. Scacchi, L.F. Pavarino, and I. Milano. Multilevel Schwarz and Multigrid preconditioners for the Bidomain system. Lecture Notes in Computational Science and Engineering, 60:631, 2008. 
[49] M. Sermesant, Ph. Moireau, O. Camara, J. Sainte-Marie, R. Andriantsimiavona, R. Cimrman, D.L. Hill, D. Chapelle, and R. Razavi. Cardiac function estimation from mri using a heart model and data assimilation: advances and difficulties. Med. Image Anal., 10(4):642-656, 2006.

[50] A.V. Shahidi, P. Savard, and R. Nadeau. Forward and inverse problems of electrocardiography: modeling and recovery of epicardial potentials in humans. IEEE Trans. Biomed. Eng., 41(3):249-256, 1994.

[51] J. Sundnes, G.T. Lines, X. Cai, B.F. Nielsen, K.-A. Mardal, and A. Tveito. Computing the electrical activity in the heart. Springer-Verlag, 2006.

[52] J. Sundnes, G.T. Lines, K.A. Mardal, and A. Tveito. Multigrid block preconditioning for a coupled system of partial differential equations modeling the electrical activity in the heart. Comput. Methods Biomech. Biomed. Engin., 5(6):397-409, 2002.

[53] A. Toselli and O. Widlund. Domain decomposition methods-algorithms and theory, volume 34 of Springer Series in Computational Mathematics. Springer-Verlag, Berlin, 2005.

[54] M.-C. Trudel, B. Dubé, M. Potse, R.M. Gulrajani, and L.J. Leon. Simulation of qrst integral maps with a membrane-based computer heart model employing parallel processing. IEEE Trans. Biomed. Eng., 51(8):1319 1329, 2004.

[55] L. Tung. A bi-domain model for describing ischemic myocardial D-C potentials. PhD thesis, MIT, 1978.

[56] F.H. van Capelle and D. Durrer. Computer simulation of arrhythmias in a network of coupled excitable elements. Circ. Res., 47:453-466, 1980.

[57] E.J. Vigmond and C. Clements. Construction of a computer model to investigate sawtooth effects in the purkinje system. IEEE Trans. Biomed. Engng., 54(3):389-399, 2007.

[58] E.J. Vigmond, R. Weber dos Santos, A.J. Prassl, M Deo, and G. Plank. Solvers for the cardiac bidomain equations. Progr. Biophys. Molec. Biol., 96(1-3):3-18, 2008.

[59] G.-X. Yan and C. Antzelevitch. Cellular basis for the normal T wave and the electrocardiographic manifestations of the long-QT syndrome. Circulation, 98:1928-1936, 1998.

\section{Contents}

\begin{tabular}{lll}
\hline & Introduction & 3
\end{tabular}

2 Modeling 4

2.1 Heart tissue . . . . . . . . . . . . . . . . . . . . . 4

2.2 Coupling with torso . . . . . . . . . . . . . . 6 
\begin{tabular}{lll}
\hline 3 & Numerical methods & 7
\end{tabular}

$3.1 \quad$ Space and time discretization $\ldots \ldots \ldots \ldots \ldots \ldots$

3.2 Partitioned heart-torso coupling. . . . . . . . . . . . . . 8

$\begin{array}{lll}4 & \text { Numerical results } & 10\end{array}$

4.1 Reference simulation . . . . . . . . . . . . . . . . . . . . . 10

4.1.1 Anatomical model and computational meshes . . . . . . . 10

4.1 .2 Heart conductivity . . . . . . . . . . . . . . . . 10

4.1 .3 Torso conductivity . . . . . . . . . . . . . . . . . 12

4.1 .4 His bundle and Purkinje fibers . . . . . . . . . . . . . 12

4.1 .5 Cell heterogeneity $\ldots \ldots \ldots \ldots$. . . . . . . . . . . . 12

4.1 .6 Results $\ldots \ldots \ldots \ldots \ldots$

4.2 Pathological simulations $\ldots \ldots \ldots \ldots$

\begin{tabular}{|lll}
5 & Impact of some modeling assumptions & 17
\end{tabular}

5.1 Heart-torso uncoupling . . . . . . . . . . . . . . . . . . . 17

5.1 .1 Numerical results . . . . . . . . . . . . . . . . . . . 18

5.1 .2 Torso transfer matrix computation . . . . . . . . . . . 20

5.2 Study of the monodomain model . . . . . . . . . . . . . . . 22

5.2 .1 The monodomain approximation . . . . . . . . . . . 22

5.2 .2 Numerical results with heart-torso uncoupling. . . . . . . 24

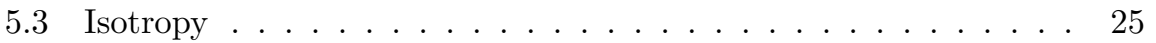

5.4 Cell homogeneity . . . . . . . . . . . . . . . . . . . . 26

$5.5 \quad$ Capacitive and resistive effect of the pericardium $\ldots \ldots \ldots 27$

6 Numerical investigations with weak heart-torso coupling $\quad 30$

6.1 Time and space convergence $\ldots \ldots \ldots \ldots \ldots$

6.1 .1 Time convergence . . . . . . . . . . . . . . . . . . 30

6.1 .2 Space convergence . . . . . . . . . . . . . . . 30

6.2 Sensitivity to model parameters . . . . . . . . . . . . . . . 31

$6.2 .1 \quad$ Ionic model parameters . . . . . . . . . . . . . . . . 32

6.2 .2 Bidomain model parameters. . . . . . . . . . . . . . . 32

6.2 .3 Torso parameters $\ldots \ldots \ldots \ldots \ldots \ldots$. . . . . . . . 34

\begin{tabular}{lll}
\hline 7 & Conclusion & 35
\end{tabular}

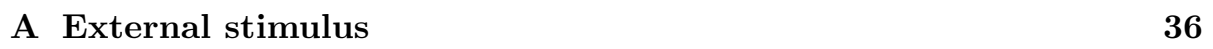


Unité de recherche INRIA Rocquencourt Domaine de Voluceau - Rocquencourt - BP 105 - 78153 Le Chesnay Cedex (France)

Unité de recherche INRIA Futurs : Parc Club Orsay Université - ZAC des Vignes 4, rue Jacques Monod - 91893 ORSAY Cedex (France)

Unité de recherche INRIA Lorraine : LORIA, Technopôle de Nancy-Brabois - Campus scientifique 615, rue du Jardin Botanique - BP 101 - 54602 Villers-lès-Nancy Cedex (France)

Unité de recherche INRIA Rennes : IRISA, Campus universitaire de Beaulieu - 35042 Rennes Cedex (France)

Unité de recherche INRIA Rhône-Alpes : 655, avenue de l'Europe - 38334 Montbonnot Saint-Ismier (France) Unité de recherche INRIA Sophia Antipolis : 2004, route des Lucioles - BP 93 - 06902 Sophia Antipolis Cedex (France) 